\title{
FIRE CLAYS; SOME FUNDAMENTAL PROPERTIES AT SEVERAL TEMPERATURES ${ }^{1}$
}

\author{
By R. A. Heindl and W. L. Pendergast
}

\section{ABSTRACT}

This report gives the results obtained in the second phase of an extensive investigation of fire-clay refractories. It is limited to the 26 representative clays included in that investigation. The geologic formation, chemical analyses, and pyrometric cone equivalents are given, together with such properties of the clays fired at two widely different temperatures, as porosity, shrinkage, thermal expansion, moduli of elasticity and rupture, and plastic flow. Data are given to show the conversion of aluminous fire clays into mullite and cristobalite by firing at certain temperatures. At higher temperatures the cristobalite is converted to glass. ?

\section{CONTENTS}

I. Introduction

II. Materials and identification 213

III. Preparation of specimens.

IV. Methods of testing

1. Chemical analyses............. 216

2. Softening point test (pyrometric cone equivalent)

3. Thermal expansion

4. Modulus of elasticity, transverse strength, and plastic flow_-- 216

5. Supplementary studies.......... 216

V. Results _..._... 217

1. Chemical analyses...... 217

2. Tempering water and absorption of grog

3. Linear shrinkage and transverse strength

4. Porosity of bodies..... 219

5. Pyrometric cone equivalents. 221

6. Linear thermal expansion 221

7. The effect of temperature of firing on the mineralogical com-

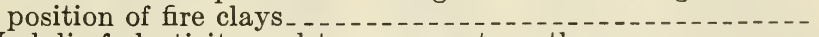

8. Moduli of elasticity and transverse strength

9. Plastic flow

10. Grouping of clays

VI. Summary

\section{INTRODUC'TION}

A report ${ }^{2}$ was published during the past year giving the results of an extended study of fire-clay bricks, together with some preliminary test data of the fire clays used in their manufacture. In order that the present paper may contain all information obtained on the clays included in the present investigation, the data previously published will appear in this report, together with the results obtained in recent studies. The chemical analyses of the clays, pyrometric cone equivalent values and such physical properties as were determined after firing them at two widely different temperatures are given.

1 This paper is the second of a series of reports which will be published on the general investigation of ire-clay refractories being conducted by this bureau.

2 Progress Report on Investigation of Fire-Clay Bricks and the Clays Used in Their Preparation, B. S. Jour. Research, 3 (RP. 114); also J. Am. Ceram, Soc., 12 (10), pp. 640-675; 1929. 


\section{MATERIALS AND IDENTIFICATION}

Twenty-six clays were furnished for this study by 12 manufacturers of refractory products. These clays were classed as follows: 7 flint, 4 semiflint, 12 plastic, and 3 miscellaneous. In addition, three samples of quartz and sand were included.

All clays supplied by any one manufacturer. were given one letter of the alphabet and also a number following this letter to identify the various clays furnished by each company. Furthermore, letter identification of the clays in each case correspond with that assigned to the respective fire-clay bricks, for example, clays $\mathrm{A}-1$ and $\mathrm{A}-2$ are the clays supplied by the manufacturer of fire-clay brick having key letter "A" as given in the preceding report."

The approximate location of the mines where the clays are produced and the geologic formation as furnished by the companies cooperating in this study and supplemented in some cases by State geologists are given briefly in Table 1.

TABLE 1.-Type of clay, origin, and geologic formation

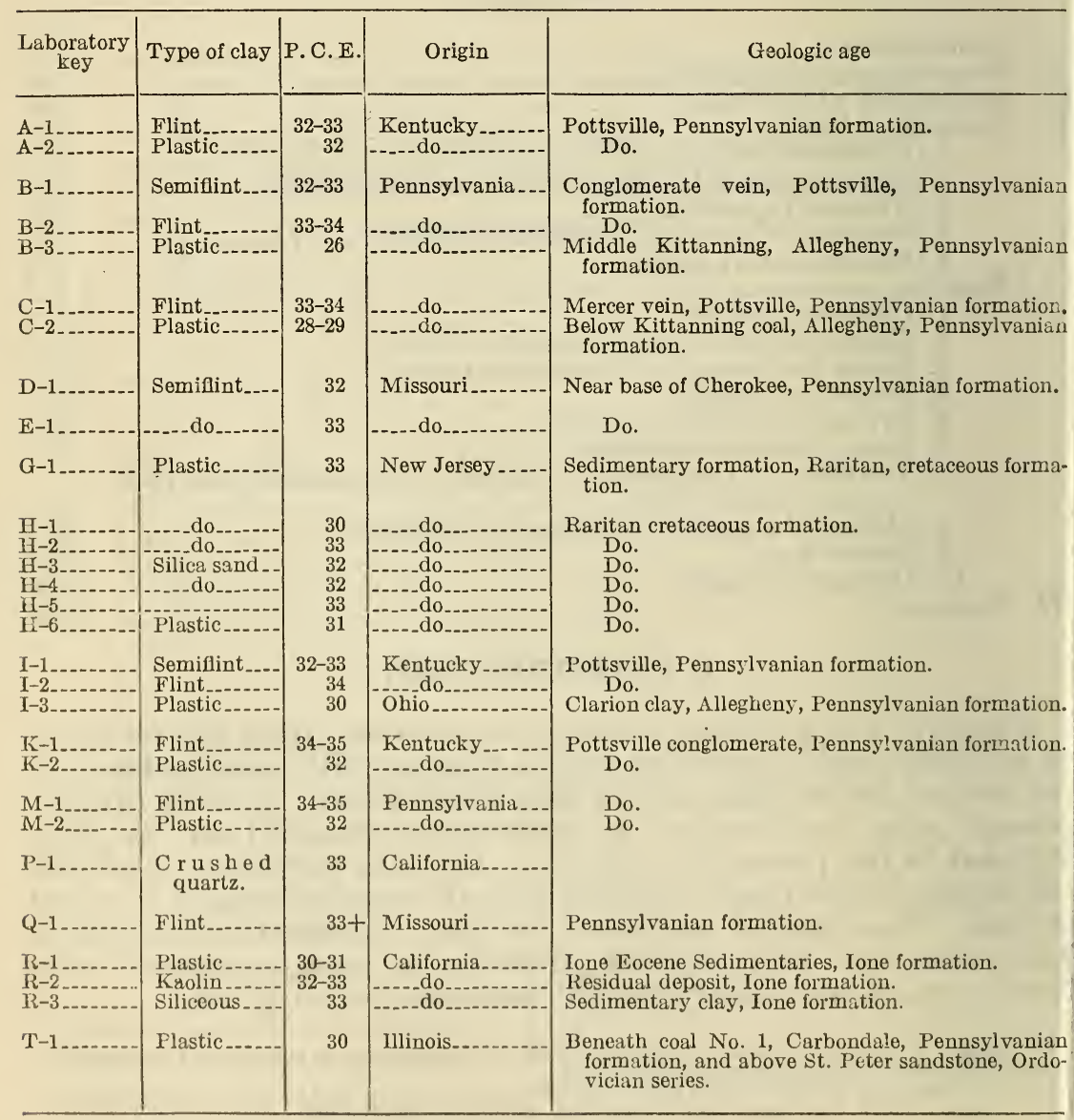

${ }^{3}$ See footnote 2, p. 213. 


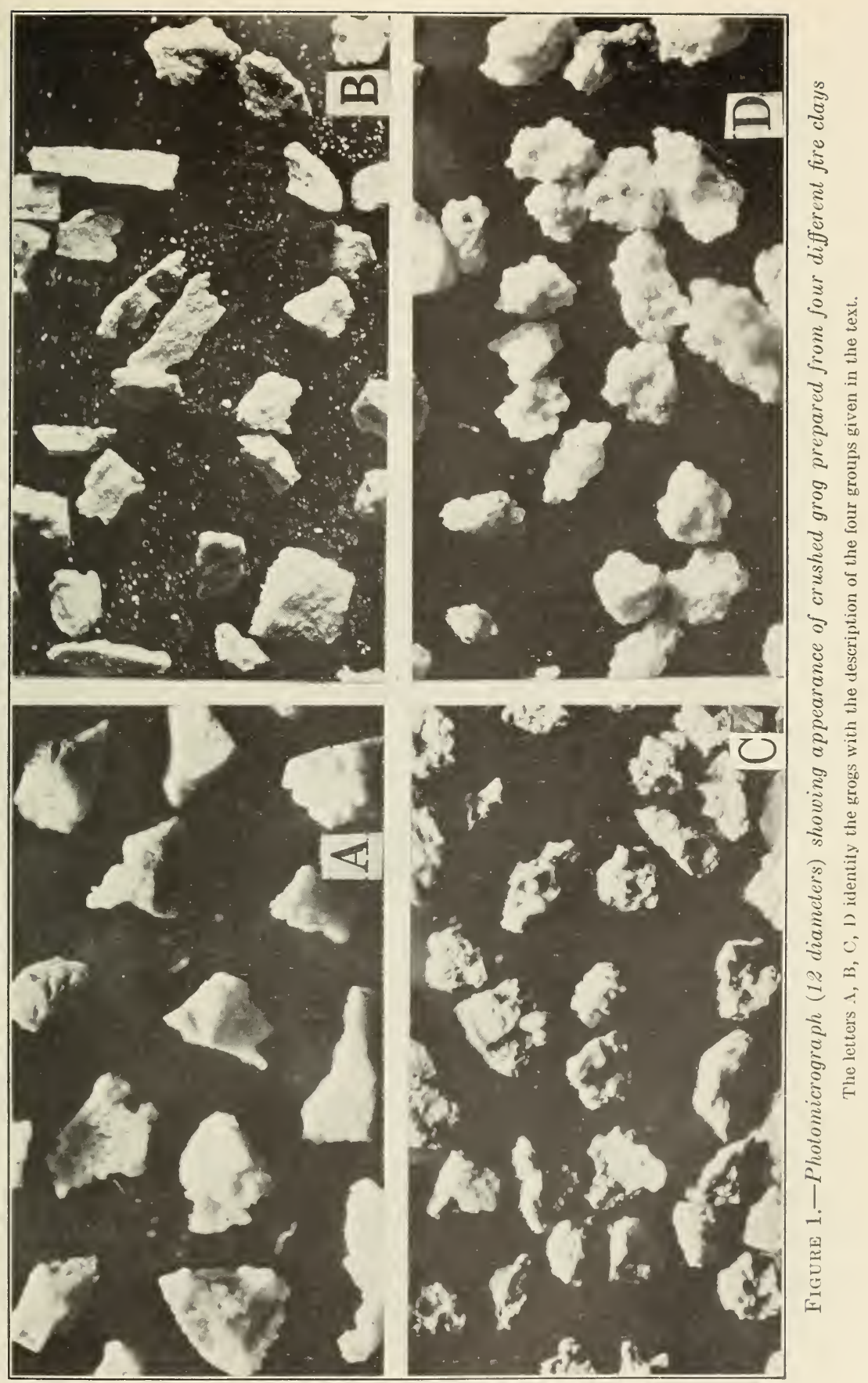




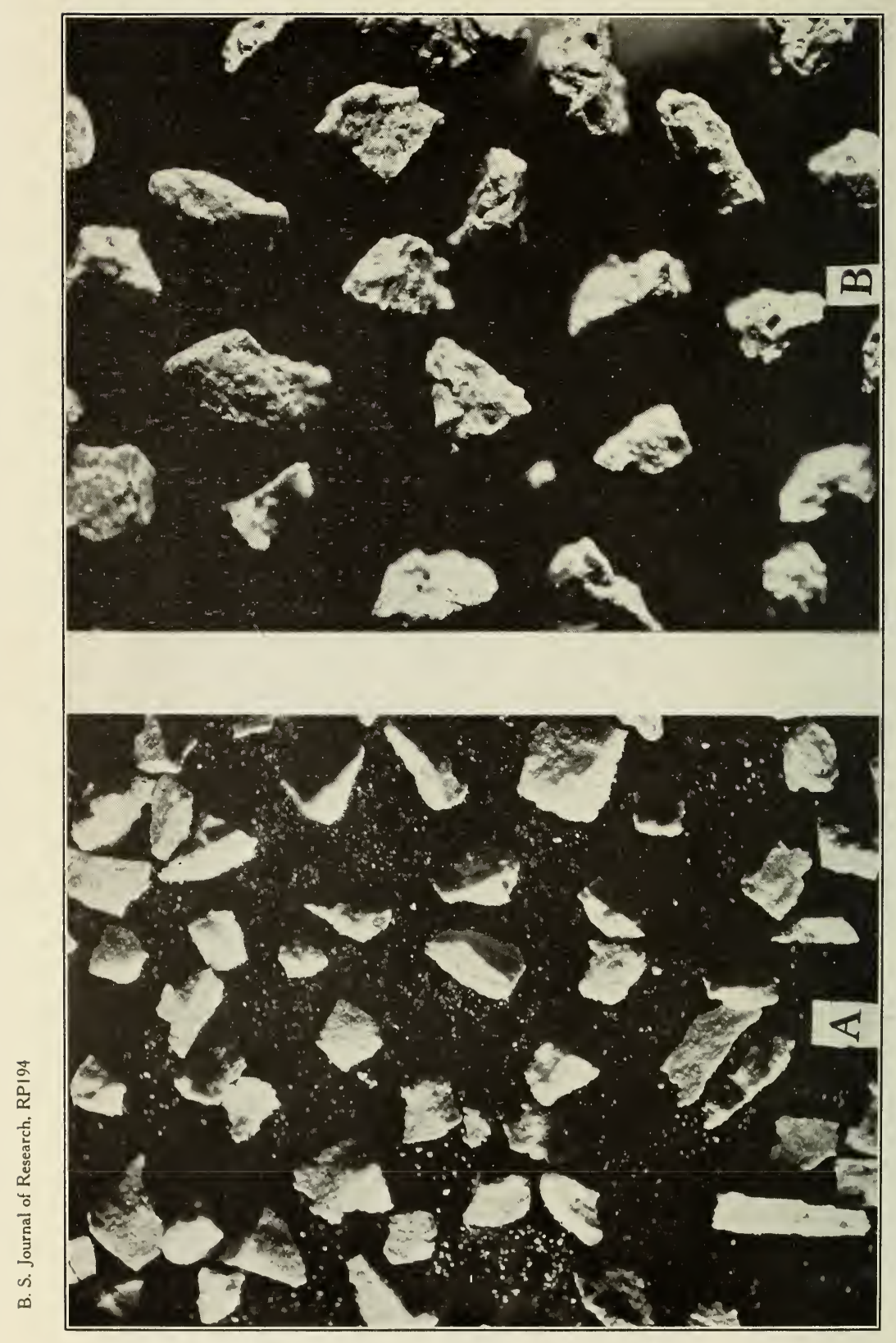

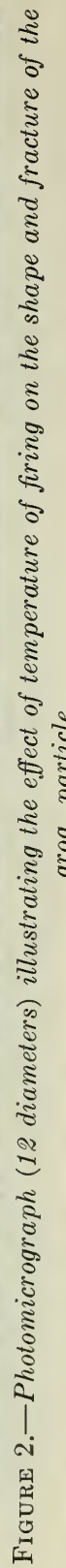




\section{PREPARATION OF SPECIMENS}

Two series of 26 different bodies were prepared. Each body contained only one raw clay and an equal amount of the same clay calcined (grog). The grog in the one series of bodies was fired at approximately $1,155^{\circ} \mathrm{C}$. for three hours (cone 4 down) and the other series contained grog fired at $1,400^{\circ} \mathrm{C}$. (cone 14 down) for the same length of time. Specimens of the clay bodies for obtaining experimental data were also fired at $1,155^{\circ} \mathrm{C}$. and $1,400^{\circ} \mathrm{C}$. for three hours. Those fired at $1,155^{\circ} \mathrm{C}$. contained the grog fired at $1,155^{\circ} \mathrm{C}$. and those fired at $1,400^{\circ} \mathrm{C}$. contained the grog fired at $1,400^{\circ} \mathrm{C}$. The grog was added as a part of the clay body in each instance to reduce warping of the specimens during drying and firing. In order to insure uniform and comparable results, the clay in each case was ground to pass a 100-mesh sieve, and the grog was crushed and graded into three sizes. The grog used in the bodies consisted of the following combination of sizes:

\begin{tabular}{|c|c|c|}
\hline \multirow{2}{*}{$\begin{array}{l}\text { Percent- } \\
\text { age of } \\
\text { each size }\end{array}$} & \multicolumn{2}{|c|}{$\begin{array}{l}\text { Sieve No. (U. S. } \\
\text { standard series) }\end{array}$} \\
\hline & Passed- & $\begin{array}{l}\text { Retained } \\
\text { on- }\end{array}$ \\
\hline $\begin{array}{l}50 \\
25 \\
25\end{array}$ & $\begin{array}{r}20 \\
40 \\
100\end{array}$ & $\begin{array}{r}40 \\
100 \\
\text { Pan. }\end{array}$ \\
\hline
\end{tabular}

An examination of the crushed grogs indicated that, according to fracture and appearance, there are four general types. The characteristics of the individual particles in some cases are not clearly defined, but most of the grogs could be classified according to the following groups: faces.

(a) More or less regular in shape with comparatively sharp cleavage

(b) More or less regular in shape with a tendency to have a platy fracture.

(c) Irregularly shaped without sharp fractures.

(d) Slightly rounded and somewhat soft.

A photomicrograph of a sample of grog representing each of the foregoing groups is given in Figure 1. The effect of temperature of firing on the fracture or cleavage and general appearance of a grog $(\mathrm{R}-1)$ is illustrated in the photomicrograph given in Figure 2.

Eight specimens, 1 by 1 by 12 inches, and six specimens, 1 by 1 by 6 inches, were prepared from each of the 52 bodies. The 12-inch specimens were used for modulus of elasticity and transverse strength determinations and the 6-inch specimens for obtaining such properties as "tempering" or "conditioning" water, linear shrinkage, and dry strength. Data referred to in this report relative to absorption of grog, chemical analyses, pyrometric cone equivalent values, and mineralogical changes of clays fired at certain temperatures were obtained on the clays alone, and data on the other properties studied were obtained on the specimens containing equal parts of clay and grog. 


\section{IMETHODS OF TESTING}

\section{CHEMICAL ANALYSES}

In making the chemical analyses of the fire clays the procedure followed was, in general, the method of analysis for refractories described by Lundell and Hoffman."

\section{SOFTENING POINT TEST (PYROMETRIC CONE EQUIVALENT)}

The softening point (P. C. E.) test was carried out according to the method approved by the bureau, and known as the A. S. T. M. standard method, serial designation C24-20. ${ }^{5}$

\section{THERMAL EXPANSION}

Linear thermal expansions of the fire clays were determined by a direct method ${ }^{6}$ on specimens measuring approximately 1 by 1 by 6 inches and which had been fired at $1,155^{\circ} \mathrm{C}$. and at $1,400^{\circ} \mathrm{C}$. Determinations were made from $20^{\circ}$ to $1,000^{\circ} \mathrm{C}$.

\section{MODULUS OF ELASTICITY, TRANSVERSE STRENGTH, AND PLASTIC FLOW}

A detailed description of the apparatus used in observing deflections of the specimens under various loads, the determination of the transverse strength (modulus of rupture), and the method for determining the load-deflection curves were published in the Third Progress Report of the Sagger Investigation. ${ }^{8}$ The modulus of elasticity and modulus of rupture were determined at several temperatures, the former from the load-deflection curve and the latter using the load which broke the specimen.

The plastic flow at $1,000^{\circ} \mathrm{C}$. of the various fire-clay bodies was determined with the same apparatus as was used to determine the elasticity and breaking strength. The plastic flow of the material under any particular stress increment was considered as the "set" or difference between the deflection caused by the load and the recovery of the material on removal of this load. A detailed description of the method for determining this property was given in the Fourth Progress Report of the Sagger Investigation. ${ }^{9}$

\section{SUPPLEMENTARY STUDIES}

Linear drying shrinkage and dry strength were determined on the 6 -inch specimens of the fire-clay bodies after drying for several days at atmospheric temperature and finally at approximately $110^{\circ} \mathrm{C}$. for 24 hours and cooling in a desicator. Linear burned shrinkage was obtained from specimens fired at $1,155^{\circ}$ and $1,400^{\circ} \mathrm{C}$. These specimens were fired at the same time as those on which the other test data were determined.

- Analysis of Bauxite and of Refractories of High Alumina Content, B. S. Jour. Research, 1 (RP5); July, 1928.

8 Described in Am. Soc. Testing Materials Standards for 1927, Pt. II, p. 151.

6 For a description of the apparatus and method used, see J. Am. Ceram. Soc., 9 (9), pp. 555-574; 1926.

7 Room temperature was approximately $20^{\circ} \mathrm{C}$. during these experiments.

8 J. Am. Ceram. Soc., 10 (7), pp. 521-534; 1927.

J. Am. Ceram. Soc., 10 (12), pp. 995-1004; 1927. 
The water porosity was determined by treating the specimens in a vacuum for one hour at an absolute pressure of $6 \mathrm{~mm}$ of mercury. Distilled water was added while the specimens were still in vacuum and then boiled in vacuum for one and one-half hours. The specimens remained under water an additional 18 hours before being weighed.

\section{RESULTS}

\section{CHEMICAL ANALYSES}

The chemical analyses of the materials investigated are given in Table 2. A study of the data shows a wide variation in composition Samples $\mathrm{H}-3, \mathrm{H}-4$, and $\mathrm{P}-1^{10}$ (Table 2) are composed largely of silica. On the other hand, fire clays $\mathrm{K}-1, \mathrm{M}-1$, and $\mathrm{Q}-1$ have a composition which closely approaches that of kaolin containing very little free quartz, and are here referred to as "aluminous."

TABLE 2.-Chemical analyses of fire clays

\begin{tabular}{|c|c|c|c|c|c|c|c|c|}
\hline $\begin{array}{c}\text { Laboratory key } \\
\text { letter }\end{array}$ & $\begin{array}{l}\text { Ignition } \\
\text { loss }\end{array}$ & $\mathrm{SiO}_{2}$ & $\mathrm{AJ}_{2} \mathrm{O}_{3}$ & $\mathrm{Fe}_{2} \mathrm{O}_{3}$ & $\mathrm{TiO}_{2}$ & $\mathrm{CaO}$ & $\mathrm{MgO}$ & Total \\
\hline $\begin{array}{l}\mathrm{A}-1 \\
\mathrm{~A}-2 \\
\mathrm{~B}-1\end{array}$ & $\begin{array}{r}\text { Per cent } \\
14.30 \\
14.08 \\
13.26 \\
14.58 \\
8.16\end{array}$ & $\begin{array}{r}\text { Per cent } \\
43.32 \\
44.64 \\
43.38 \\
43.06 \\
58.68\end{array}$ & $\begin{array}{r}\text { Per cent } \\
35.72 \\
35.84 \\
37.97 \\
37.84 \\
24.95\end{array}$ & $\begin{array}{r}\text { Per cent } \\
3.26 \\
2.39 \\
1.39 \\
2.14 \\
2.39\end{array}$ & $\begin{array}{r}\text { Per cent } \\
2.02 \\
1.77 \\
2.12 \\
1.84 \\
1.70\end{array}$ & $\begin{array}{r}\text { Per cent } \\
0.18 \\
.26 \\
.12 \\
.19\end{array}$ & $\begin{array}{r}\text { Per cent } \\
0.40 \\
.52 \\
.31 \\
.04 \\
.70\end{array}$ & $\begin{array}{r}\text { Per cent } \\
99.20 \\
99.50 \\
98.43 \\
99.62 \\
96.77\end{array}$ \\
\hline $\begin{array}{l}\mathrm{C}-1 \\
\mathrm{C}-2 \\
\mathrm{D}-1 \\
\mathrm{E}-1\end{array}$ & $\begin{array}{l}13.78 \\
10.86 \\
12.34 \\
14.06 \\
14.32\end{array}$ & $\begin{array}{l}42.74 \\
50.38 \\
51.8 ? \\
45.98 \\
47.09\end{array}$ & $\begin{array}{l}39.18 \\
30.87 \\
32.09 \\
37.12 \\
36.25\end{array}$ & $\begin{array}{l}1.01 \\
2.56 \\
1.24 \\
.88 \\
.75\end{array}$ & $\begin{array}{l}2.33 \\
2.27 \\
1.63 \\
1.90 \\
1.89\end{array}$ & .32 & $\begin{array}{l}.29 \\
.30 \\
.58 \\
.29 \\
.06\end{array}$ & $\begin{array}{r}99.33 \\
97.56 \\
99.70 \\
100.35 \\
100.80\end{array}$ \\
\hline $\begin{array}{l}\mathrm{H}-1 . \\
\mathrm{H}-2 . \\
\mathrm{H}-3 . \\
\mathrm{H}-4 \\
\mathrm{H}-5 .\end{array}$ & $\begin{array}{r}7.42 \\
14.36 \\
1.62 \\
2.86 \\
12.82\end{array}$ & $\begin{array}{l}70.29 \\
46.36 \\
91.52 \\
87.68 \\
52.26\end{array}$ & $\begin{array}{r}19.46 \\
35.43 \\
4.65 \\
7.33 \\
32.30\end{array}$ & $\begin{array}{r}.88 \\
1.87 \\
.28 \\
1.06 \\
.84\end{array}$ & $\begin{array}{l}2.02 \\
1.57 \\
.67 \\
1.25 \\
1.72\end{array}$ & $\begin{array}{l}.22 \\
.44 \\
.26 \\
.38 \\
.26\end{array}$ & $\begin{array}{l}.03 \\
.46 \\
.31 \\
.14 \\
.02\end{array}$ & $\begin{array}{r}100.32 \\
100.50 \\
99.31 \\
100.70 \\
100.02\end{array}$ \\
\hline $\begin{array}{l}\mathrm{H}-6 \\
\mathrm{I}-1-2 \\
\mathrm{I}-2\end{array}$ & $\begin{array}{r}8.14 \\
11.62 \\
13.66 \\
10.62 \\
14.86\end{array}$ & $\begin{array}{l}68.20 \\
51.42 \\
44.66 \\
53.50 \\
42.50\end{array}$ & $\begin{array}{l}21.37 \\
32.80 \\
37.58 \\
29.82 \\
39.68\end{array}$ & $\begin{array}{r}.78 \\
.93 \\
1.33 \\
1.95 \\
.76\end{array}$ & $\begin{array}{l}1.63 \\
2.33 \\
1.53 \\
1.73 \\
2.34\end{array}$ & $\begin{array}{l}.34 \\
.66 \\
.23\end{array}$ & $\begin{array}{l}.12 \\
.52 \\
.15 \\
.16 \\
.12\end{array}$ & $\begin{array}{r}100.58 \\
99.62 \\
99.67 \\
98.01 \\
100.26\end{array}$ \\
\hline $\begin{array}{l}\mathrm{K}-2 \\
\mathrm{M}-1 \\
\mathrm{M}-2 \mathrm{C} \\
\mathrm{P}-1\end{array}$ & $\begin{array}{r}12.28 \\
13.68 \\
11.46 \\
.28 \\
13.32\end{array}$ & $\begin{array}{l}47.00 \\
44.56 \\
45.76 \\
97.48 \\
42.32\end{array}$ & $\begin{array}{l}33.41 \\
38.19 \\
35.67 \\
.72 \\
39.63\end{array}$ & $\begin{array}{r}2.59 \\
1.12 \\
1.95 \\
.14 \\
.84\end{array}$ & $\begin{array}{l}2.00 \\
2.21 \\
1.72 \\
.46 \\
1.81\end{array}$ & $\begin{array}{l}.48 \\
.14 \\
.30 \\
.16 \\
.68\end{array}$ & $\begin{array}{l}.32 \\
.10 \\
.64 \\
.32 \\
.50\end{array}$ & $\begin{array}{r}98.08 \\
100.00 \\
97.50 \\
99.56 \\
99.10\end{array}$ \\
\hline $\begin{array}{l}R-1 \\
R-2 \\
R-3 \\
T-1\end{array}$ & $\begin{array}{r}12.06 \\
14.66 \\
7.22 \\
10.92\end{array}$ & $\begin{array}{l}52.78 \\
44.46 \\
72.78 \\
60.26\end{array}$ & $\begin{array}{l}29.90 \\
37.31 \\
18.94 \\
25.28\end{array}$ & $\begin{array}{l}2.37 \\
1.13 \\
.65 \\
1.62\end{array}$ & $\begin{array}{l}1.07 \\
2.76 \\
.61 \\
1.28\end{array}$ & $\begin{array}{l}.04 \\
.20 \\
.23 \\
.38\end{array}$ & $\begin{array}{r}1.20 \\
.12 \\
.14 \\
.16\end{array}$ & $\begin{array}{r}99.42 \\
100.64 \\
100.57 \\
99.90\end{array}$ \\
\hline
\end{tabular}

\section{TEMPERING WATER AND ABSORPTION OF GROG}

The tempering water-that is, the percentage of water considered as desirable for securing proper molding consistency of the clay test specimens-is given in Table 3 . This information is given for those specimens containing grog fired to cone 4 as well as for those containing grog fired to cone 14 .

${ }_{10}$ Complete test data on materials $\mathrm{H}-3, \mathrm{H}-4$, and $\mathrm{P}-1$ were not obtained because they are largely silica. 
The percentage absorption of the grog fired at the two temperatures is also given in Table 3. The data on absorption show only two clays (B-3 and C-2) overfired at cone 14 . It may be that other clays are overfired, but this is not indicated by their appearance, and since no burns were made between cones 4 and 14, it is not known whether any of the clays reached an absorption lower than that given in the table. The smallest change in absorption was shown by the grog prepared from the highly siliceous clays.

TABLE 3.-Tempering water of fire clay bodies and absorption of grogs used

\begin{tabular}{|c|c|c|c|c|}
\hline \multirow[t]{2}{*}{ Laboratory key } & \multicolumn{2}{|c|}{$\begin{array}{l}\text { Tempering water; } \\
\text { specimens con- } \\
\text { taining grog fired } \\
\text { to- }\end{array}$} & \multicolumn{2}{|c|}{$\begin{array}{l}\text { Absorption (per } \\
\text { unit weight); grog } \\
\text { fired to- }\end{array}$} \\
\hline & Cone 4 & Cone 14 & Cone 4 & Cone 14 \\
\hline $\begin{array}{l}A-1 \\
A-2\end{array}$ & $\begin{array}{r}\text { Per cent } \\
17.3 \\
18.1\end{array}$ & $\begin{array}{r}\text { Per cent } \\
16.1 \\
16.0\end{array}$ & $\begin{array}{r}\text { Per cent } \\
11.6 \\
10.6\end{array}$ & $\begin{array}{r}\text { Per cent } \\
9.4 \\
.9\end{array}$ \\
\hline 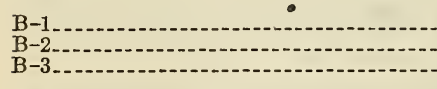 & $\begin{array}{l}18.1 \\
18.9 \\
17.3\end{array}$ & $\begin{array}{l}19.1 \\
17.4 \\
16.1\end{array}$ & $\begin{array}{r}12.3 \\
16.7 \\
7.8\end{array}$ & $\begin{array}{r}7.4 \\
1.1 \\
12.0\end{array}$ \\
\hline $\begin{array}{l}\mathrm{C}-1 \\
\mathrm{C}-2-(-2-1\end{array}$ & $\begin{array}{l}21.4 \\
16.5\end{array}$ & $\begin{array}{l}16.7 \\
16.7\end{array}$ & $\begin{array}{r}16.0 \\
6.0\end{array}$ & $\begin{aligned} 11.6 & \\
8.7 & -1\end{aligned}$ \\
\hline $\begin{array}{l}\mathrm{D}-1 \\
\mathrm{E}-1-1 .\end{array}$ & $\begin{array}{l}18.1 \\
18.8 \\
23.9\end{array}$ & $\begin{array}{l}14.5 \\
14.9 \\
16.9\end{array}$ & $\begin{array}{r}7.7 \\
13.8 \\
26.3\end{array}$ & $\begin{array}{l}3.6 \\
2.6 \\
6.3\end{array}$ \\
\hline $\begin{array}{l}\mathrm{H}-1 \\
\mathrm{H}-2 \\
\mathrm{H}-5 \\
\mathrm{H}-6\end{array}$ & $\begin{array}{l}20.9 \\
22.0 \\
24.8 \\
20.1\end{array}$ & $\begin{array}{l}19.7 \\
19.3 \\
18.4 \\
19.7\end{array}$ & $\begin{array}{r}20.7 \\
9.6 \\
20.9 \\
20.0\end{array}$ & $\begin{array}{r}18.1 \\
2.6 \\
67.8 \\
17.5\end{array}$ \\
\hline I-1-2 & $\begin{array}{l}18.1 \\
18.1 \\
18.7\end{array}$ & $\begin{array}{l}14.8 \\
14.8 \\
15.1\end{array}$ & $\begin{array}{l}13.0 \\
14.6 \\
11.5\end{array}$ & $\begin{array}{l}6.8 \\
6.7 \\
1.1\end{array}$ \\
\hline $\begin{array}{l}\mathrm{K}-1 \\
\mathrm{~K}-2-2\end{array}$ & $\begin{array}{l}20.5 \\
19.4\end{array}$ & $\begin{array}{l}16.6 \\
17.2\end{array}$ & $\begin{array}{r}17.6 \\
7.9\end{array}$ & $\begin{array}{r}11.1 \\
.4\end{array}$ \\
\hline $\begin{array}{l}M-1 \\
M-2\end{array}$ & $\begin{array}{l}20.8 \\
19.2\end{array}$ & $\begin{array}{l}18.1 \\
17.2\end{array}$ & $\begin{array}{l}19.7 \\
22.3\end{array}$ & $\begin{array}{r}11.7 \\
6.9\end{array}$ \\
\hline Q-1... & 18.1 & 16.6 & 10.7 & 4.5 \\
\hline $\begin{array}{l}\mathrm{R}-1 \mathrm{-1}-\mathrm{-} \\
\mathrm{R}-2 \mathrm{-} \\
\mathrm{R}-3 .\end{array}$ & $\begin{array}{l}20.1 \\
22.3 \\
26.8\end{array}$ & $\begin{array}{l}19.4 \\
18.3 \\
28.5\end{array}$ & $\begin{array}{r}10.4 \\
9.2 \\
26.1\end{array}$ & $\begin{array}{r}5.5 \\
1.4 \\
22.5\end{array}$ \\
\hline T-1 - & 16.4 & 15. 3 & 7.9 & 6.5 \\
\hline
\end{tabular}

The absorption of the grog apparently has little effect on the amount of tempering water used in preparing the specimens. Clay B-2 may be cited as a typical example. The percentage absorption decreased approximately 15.5 between the cone 4 and cone 14 firing, but the difference in percentage tempering water was only 1.5 .

\section{LINEAR SHRINKAGE AND TRANSVERSE STRENGTH}

The linear shrinkage and strength after drying and the total linear shrinkage after firing of the specimens prepared with grog fired at the two different temperatures are given in Table 4. 
TABLE 4.-Dry and fired shrinkage, and strength of fire-clay bodies

\begin{tabular}{|c|c|c|c|c|c|c|}
\hline \multirow{3}{*}{ Laboratory key } & \multicolumn{2}{|c|}{$\begin{array}{l}\text { Linear drying } \\
\text { shrinkage }\end{array}$} & \multicolumn{2}{|c|}{$\begin{array}{l}\text { Total fired } \\
\text { shrinkage }\end{array}$} & \multicolumn{2}{|c|}{$\begin{array}{c}\text { Dry strength (cross } \\
\text { breaking) }\end{array}$} \\
\hline & \multicolumn{6}{|c|}{ Specimens containing grog fired to- } \\
\hline & Cone 4 & Cone 14 & Cone 4 & Cone 14 & Cone 4 & Cone 14 \\
\hline$A-1, \ldots$ & $\begin{array}{r}\text { Per cent } \\
3.7 \\
3.4\end{array}$ & $\begin{array}{r}\text { Per cent } \\
4.4 \\
4.6\end{array}$ & $\begin{array}{r}\text { Per cent } \\
8.3 \\
7.2\end{array}$ & $\begin{array}{r}\text { Per cent } \\
7.3 \\
8.3\end{array}$ & $\begin{array}{r}\text { Lbs./in } .^{2} \\
225 \\
155\end{array}$ & $\begin{array}{r}\text { Lbs./in. }{ }^{2} \\
145 \\
145\end{array}$ \\
\hline $\begin{array}{l}\mathrm{B}-1 \\
\mathrm{~B}-2 \\
\mathrm{~B}-3\end{array}$ & $\begin{array}{l}3.0 \\
2.6 \\
3.0\end{array}$ & $\begin{array}{l}3.2 \\
4.9 \\
3.8\end{array}$ & $\begin{array}{l}6.5 \\
5.0 \\
9.0\end{array}$ & $\begin{array}{r}12.5 \\
6.9 \\
6.9\end{array}$ & $\begin{array}{r}90 \\
60 \\
140\end{array}$ & $\begin{array}{r}80 \\
70 \\
150\end{array}$ \\
\hline C-1 -2 & $\begin{array}{l}\text { 3. } 1 \\
\text { 3. } 1\end{array}$ & $\begin{array}{l}5.6 \\
3.8\end{array}$ & $\begin{array}{l}6.7 \\
8.0\end{array}$ & $\begin{array}{l}7.9 \\
8.5\end{array}$ & $\begin{array}{r}55 \\
205\end{array}$ & $\begin{array}{r}60 \\
245\end{array}$ \\
\hline $\begin{array}{l}\mathrm{D}-1 \\
\mathrm{E}-1\end{array}$ & $\begin{array}{l}3.6 \\
3.2 \\
2.8\end{array}$ & $\begin{array}{l}4.0 \\
3.8 \\
3.6\end{array}$ & $\begin{array}{l}7.3 \\
8.9 \\
9.3\end{array}$ & $\begin{array}{l}7.4 \\
7.4 \\
6.6\end{array}$ & $\begin{array}{l}195 \\
190 \\
120\end{array}$ & $\begin{array}{l}195 \\
165 \\
140\end{array}$ \\
\hline $\begin{array}{l}\mathrm{H}-1 \\
\mathrm{H}-\mathrm{H}-\mathrm{H}-\mathrm{H} \\
\mathrm{H}-\mathrm{5}-\mathrm{w}\end{array}$ & $\begin{array}{l}3.0 \\
3.4 \\
3.2 \\
2.4\end{array}$ & $\begin{array}{l}4.4 \\
4.2 \\
4.2 \\
4.2\end{array}$ & $\begin{array}{l}4.3 \\
9.7 \\
8.5 \\
4.6\end{array}$ & $\begin{array}{l}2.2 \\
4.3 \\
3.7 \\
2.3\end{array}$ & $\begin{array}{r}100 \\
165 \\
190 \\
85\end{array}$ & $\begin{array}{l}135 \\
200 \\
140 \\
120\end{array}$ \\
\hline 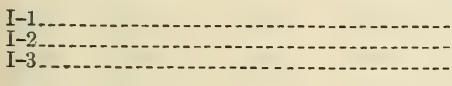 & $\begin{array}{l}2.7 \\
3.5 \\
3.9\end{array}$ & $\begin{array}{l}4.4 \\
4.8 \\
4.6\end{array}$ & $\begin{array}{l}5.5 \\
5.8 \\
8.8\end{array}$ & $\begin{array}{l}7.7 \\
6.9 \\
7.9\end{array}$ & $\begin{array}{l}110 \\
180 \\
225\end{array}$ & $\begin{array}{l}110 \\
105 \\
215\end{array}$ \\
\hline $\mathrm{K}-1,2$ & $\begin{array}{l}3.9 \\
4.2\end{array}$ & $\begin{array}{l}5.2 \\
5.6\end{array}$ & $\begin{array}{l}8.1 \\
9.2\end{array}$ & $\begin{array}{l}7.1 \\
9.2\end{array}$ & $\begin{array}{r}60 \\
180\end{array}$ & $\begin{array}{r}70 \\
225\end{array}$ \\
\hline $\begin{array}{l}M-1 \\
M-2 \ldots\end{array}$ & $\begin{array}{l}3.0 \\
3.5\end{array}$ & $\begin{array}{l}6.8 \\
4.8\end{array}$ & $\begin{array}{l}6.7 \\
9.1\end{array}$ & $\begin{array}{l}8.6 \\
9.5\end{array}$ & $\begin{array}{l}55 \\
80\end{array}$ & $\begin{array}{r}80 \\
105\end{array}$ \\
\hline Q-1.. & 4. 2 & 4.8 & 8.7 & 9.3 & 180 & 180 \\
\hline $\begin{array}{l}\mathrm{R}-1 \\
\mathrm{R}-2 \\
\mathrm{R}-3\end{array}$ & $\begin{array}{l}4.2 \\
2.5 \\
3.5\end{array}$ & $\begin{array}{l}5.4 \\
3.8 \\
6.0\end{array}$ & $\begin{array}{r}9.8 \\
13.1 \\
7.4\end{array}$ & $\begin{array}{l}9.7 \\
7.5 \\
7.9\end{array}$ & $\begin{array}{r}180 \\
85 \\
35\end{array}$ & $\begin{array}{r}375 \\
125 \\
70\end{array}$ \\
\hline T-1. & 3.4 & 5.4 & 5.3 & 6.3 & 400 & 295 \\
\hline
\end{tabular}

The majority of specimens containing grog fired at the higher temperature show greater dry strength in comparison to those containing grog fired to the lower temperature, but the average values do not show a significant difference. The data on fired shrinkage do not indicate that any of the bodies are overfired at $1,400^{\circ} \mathrm{C}$. The bodies made up of clays $\mathrm{H}-1, \mathrm{H}-5$, and $\mathrm{H}-6$, however, show a negative shrinkage (expansion) which is due to their high silica content and represents the conversion of the quartz to cristobalite. As in the case of the porosity determinations the data obtained on fired shrinkage are quite misleading as an indication of whether the bodies are overfired because it is not known whether they reached their minimum shrinkage at some temperature before $1,400^{\circ} \mathrm{C}$. In the case of body $\mathrm{B}-3$, an examination shows it to be overfired, although the shrinkage measurements do not so indicate.

\section{POROSITY OF BODIES}

The porosities of the fired specimens were determined on sections cut from the ruptured 12-inch bars on which data were obtained for moduli of elasticity and rupture computations. The results obtained 
on specimens fired at $1,155^{\circ} \mathrm{C}$. are given in Table 5 and those fired at $1,400^{\circ} \mathrm{C}$. in Table 6 . The porosity values given represent the average of not less than three determinations.

TABLE 5.-Modulus of elasticity, transverse strength, and porosity of fire clays fired at $1,155^{\circ} \mathrm{C}$. for three hours

\begin{tabular}{|c|c|c|c|c|c|c|c|}
\hline \multirow{3}{*}{ Laboratory key letter } & \multirow{2}{*}{\multicolumn{2}{|c|}{$\frac{\text { Tested at } 20^{\circ} \mathrm{C} .}{\text { Modulus of- }}$}} & \multirow{2}{*}{\multicolumn{2}{|c|}{$\frac{\text { Tested at } 550^{\circ} \mathrm{C} .}{\text { Modulus of- }}$}} & \multirow{2}{*}{\multicolumn{2}{|c|}{ Tested at $1,000^{\circ} \mathrm{C}}}$. & \multirow{3}{*}{ Porosity } \\
\hline & & & & & & & \\
\hline & Rupture & $\begin{array}{c}\text { Elastic- } \\
\text { ity }\end{array}$ & Rupture & $\begin{array}{c}\text { Elastic- } \\
\text { ity }\end{array}$ & Rupture & $\underset{\text { ity }}{\text { Elastic- }}$ & \\
\hline $\begin{array}{l}A-1 \\
A-2\end{array}$ & $\begin{array}{r}\text { Lbs./in. }{ }^{2} \\
2,755 \\
2,415\end{array}$ & $\begin{array}{r}1,000 \\
\text { lbs./in.2 } \\
5,530 \\
3,840\end{array}$ & $\begin{array}{r}\text { Lbs./in. }{ }^{2} \\
2,745 \\
2,070\end{array}$ & $\begin{array}{r}1,000 \\
\text { lbs./in. } .^{2} \\
5,275 \\
3,785\end{array}$ & $\begin{array}{r}\text { Lbs./in. }{ }^{2} \\
3,925 \\
2,110\end{array}$ & $\begin{array}{r}1,000 \\
\text { lbs./in. } .^{2} \\
1,040 \\
595\end{array}$ & $\begin{array}{r}\text { Per cent } \\
23.9 \\
27.5\end{array}$ \\
\hline $\begin{array}{l}\text { B-1 } \\
\text { B-2.-. } \\
\text { B-3... }\end{array}$ & $\begin{array}{r}1,690 \\
480 \\
3,370\end{array}$ & $\begin{array}{r}3,000 \\
835 \\
7,625\end{array}$ & $\begin{array}{r}2,055 \\
580 \\
3,555\end{array}$ & $\begin{array}{l}4,490 \\
1,270 \\
7,540\end{array}$ & $\begin{array}{l}1,950 \\
375 \\
(1)\end{array}$ & $\begin{array}{l}505 \\
205 \\
660\end{array}$ & $\begin{array}{r}28.5 \\
35.1 \\
9.2\end{array}$ \\
\hline $\begin{array}{l}\mathrm{C}-1 .- \\
\mathrm{C}-2 .-\end{array}$ & $\begin{array}{r}825 \\
3,220\end{array}$ & $\begin{array}{l}1,515 \\
7,135\end{array}$ & $\begin{array}{r}790 \\
2,775\end{array}$ & $\begin{array}{l}1,635 \\
5,610\end{array}$ & $(1)^{650}$ & $\begin{array}{l}305 \\
270\end{array}$ & $\begin{array}{l}35.4 \\
20.3\end{array}$ \\
\hline D-1_ & 2,435 & 3,945 & 2,415 & 4,200 & 2,940 & 945 & 25.5 \\
\hline E-1. & 3,205 & 5,115 & 3,250 & 6,260 & 3,240 & 1,610 & 21.2 \\
\hline $\mathrm{G}-1$ & 1,320 & 1,890 & 2,100 & 2,525 & 2,760 & 1,040 & 33.3 \\
\hline $\begin{array}{l}\mathrm{H}-1=- \\
\mathrm{H}-2-- \\
\mathrm{H}-5= \\
\mathrm{H}-6 . .\end{array}$ & $\begin{array}{r}565 \\
2,010 \\
1,100 \\
465\end{array}$ & $\begin{array}{r}584 \\
3,575 \\
1,535 \\
410\end{array}$ & $\begin{array}{r}795 \\
2,655 \\
1,640 \\
675\end{array}$ & $\begin{array}{r}1,075 \\
4,905 \\
2,020 \\
840\end{array}$ & $\begin{array}{r}925 \\
3,290 \\
1,610 \\
740\end{array}$ & $\begin{array}{r}555 \\
1,010 \\
905 \\
415\end{array}$ & $\begin{array}{l}37.4 \\
29.1 \\
35.5 \\
37.2\end{array}$ \\
\hline $\begin{array}{l}I-1 \\
I-2 \\
I-3\end{array}$ & $\begin{array}{r}1,185 \\
945 \\
2,570\end{array}$ & $\begin{array}{l}1,725 \\
2,000 \\
5,005\end{array}$ & $\begin{array}{l}1,485 \\
1,010 \\
3,160\end{array}$ & $\begin{array}{l}2,785 \\
2,080 \\
5,385\end{array}$ & $\begin{array}{r}2,160 \\
850 \\
4,300\end{array}$ & $\begin{array}{l}535 \\
295 \\
715\end{array}$ & $\begin{array}{l}30.3 \\
32.7 \\
20.4\end{array}$ \\
\hline $\begin{array}{l}\mathrm{K}-1 \text { - } \\
\mathrm{K}-2 \mathrm{-}\end{array}$ & $\begin{array}{r}825 \\
2,710\end{array}$ & $\begin{array}{l}1,650 \\
6,075\end{array}$ & $\begin{array}{r}820 \\
2,385\end{array}$ & $\begin{array}{l}1,690 \\
5,595\end{array}$ & $\begin{array}{r}825 \\
2,770\end{array}$ & $\begin{array}{l}800 \\
400\end{array}$ & $\begin{array}{l}31.4 \\
24.7\end{array}$ \\
\hline $\begin{array}{l}M-1 \\
M-2\end{array}$ & $\begin{array}{r}870 \\
2,795\end{array}$ & $\begin{array}{l}1,695 \\
5,700\end{array}$ & $\begin{array}{l}1,040 \\
2,690\end{array}$ & $\begin{array}{l}1,855 \\
5,705\end{array}$ & $\begin{array}{r}990 \\
3,665\end{array}$ & $\begin{array}{l}245 \\
465\end{array}$ & $\begin{array}{l}33.1 \\
24.7\end{array}$ \\
\hline Q-1_--. & 2,235 & 4,310 & 2,500 & 4,390 & 1,480 & 620 & 27.3 \\
\hline $\begin{array}{l}R-1= \\
R-2= \\
R-3=\end{array}$ & $\begin{array}{r}2,450 \\
2,075 \\
490\end{array}$ & $\begin{array}{r}4,730 \\
4,305 \\
505\end{array}$ & $\begin{array}{r}2,645 \\
2,335 \\
680\end{array}$ & $\begin{array}{r}5,675 \\
4,550 \\
760\end{array}$ & $\begin{array}{l}4,015 \\
2,390 \\
1,100\end{array}$ & $\begin{array}{r}730 \\
1,515 \\
590\end{array}$ & $\begin{array}{l}25.9 \\
22.0 \\
41.2\end{array}$ \\
\hline T-1. & 1,835 & 2,595 & 1,950 & 3,515 & 3,140 & 1,180 & 28.0 \\
\hline
\end{tabular}

1 Specimen did not rupture. 
TABLE 6.-Modulus of elasticity, transverse strength, and porosity of fire clays fired at $1,400^{\circ}$ C. for three hours

\begin{tabular}{|c|c|c|c|c|c|c|c|}
\hline \multirow{3}{*}{ Laboratory key letter } & \multirow{2}{*}{\multicolumn{2}{|c|}{$\frac{\text { Tested at } 20^{\circ} \mathrm{C} .}{\text { Modulus of }}$}} & \multirow{2}{*}{\multicolumn{2}{|c|}{$\frac{\text { Tested at } 550^{\circ} \mathrm{C} .}{\text { Modulus of }-}$}} & \multirow{2}{*}{\multicolumn{2}{|c|}{$\frac{\text { Tested at } 1,000^{\circ} \mathrm{C} .}{\text { Modulus n- }}$}} & \multirow{3}{*}{ Porosity } \\
\hline & & & & & & & \\
\hline & Rupture & $\begin{array}{c}\text { Elastic- } \\
\text { ity }\end{array}$ & Rupture & $\begin{array}{c}\text { Elastic- } \\
\text { ity }\end{array}$ & Rupture & $\begin{array}{c}\text { Elastic- } \\
\text { ity }\end{array}$ & \\
\hline $\begin{array}{l}A-1 \ldots \\
A-2-. .\end{array}$ & $\begin{array}{r}\text { Lbs./in. }{ }^{2} \\
2,750 \\
2,765\end{array}$ & $\begin{array}{r}1,000 \\
\text { lbs./in.2 } \\
5,800 \\
6,570\end{array}$ & $\begin{array}{r}\text { Lbs./in. }{ }^{2} \\
2,780 \\
2,745\end{array}$ & $\begin{array}{r}1,000 \\
\text { lbs./in. } \\
6,180 \\
7,415\end{array}$ & $\begin{array}{r}\text { Lbs./in. }{ }^{2} \\
3,060 \\
3,580\end{array}$ & $\begin{array}{r}1,000 \\
\text { lbs./in. } \\
1,930 \\
1,425\end{array}$ & $\begin{array}{r}\text { Per cent } \\
24.7 \\
19.7\end{array}$ \\
\hline $\begin{array}{l}\text { B-1-.- } \\
\text { B-2--: } \\
\text { B-3.-- }\end{array}$ & $\begin{array}{r}2,160 \\
635 \\
2,285\end{array}$ & $\begin{array}{l}5,060 \\
1,485 \\
6,720\end{array}$ & $\begin{array}{r}2,200 \\
725 \\
3,240\end{array}$ & $\begin{array}{l}5,320 \\
1,865 \\
8,470\end{array}$ & $\begin{array}{r}2,250 \\
705 \\
2,750\end{array}$ & $\begin{array}{l}940 \\
645 \\
300\end{array}$ & $\begin{array}{r}24.7 \\
31.1 \\
.7\end{array}$ \\
\hline $\begin{array}{l}\text { C-1 } \\
\text { C-2.... }\end{array}$ & $\begin{array}{l}1,060 \\
2,630\end{array}$ & $\begin{array}{l}2,290 \\
8,175\end{array}$ & $\begin{array}{l}2,325 \\
3,000\end{array}$ & $\begin{array}{l}2,745 \\
8,785\end{array}$ & $\begin{array}{l}1,585 \\
5,150\end{array}$ & $\begin{array}{l}990 \\
850\end{array}$ & $\begin{array}{l}25.6 \\
11.1\end{array}$ \\
\hline D-1. & 3,625 & 7,420 & 2,985 & 7,960 & 3,590 & 2,655 & 16.8 \\
\hline E-1.. & 2,240 & 5,820 & 3,190 & 7,015 & 2,800 & 2,410 & 21.8 \\
\hline G-1. & 1,960 & 3,090 & 2,170 & 4,025 & 2,295 & 2,160 & 27.4 \\
\hline $\begin{array}{l}\mathrm{H}-1 . \\
\mathrm{H}-2 . \\
\mathrm{H}-5 . \\
\mathrm{H}-6 .\end{array}$ & $\begin{array}{r}670 \\
2,745 \\
2,200 \\
695\end{array}$ & $\begin{array}{r}795 \\
6,980 \\
3,310 \\
695\end{array}$ & $\begin{array}{l}1,020 \\
2,995 \\
2,080 \\
880\end{array}$ & $\begin{array}{l}1,730 \\
7,220 \\
3,645 \\
1,335\end{array}$ & $\begin{array}{l}1,575 \\
3,120 \\
2,545 \\
1,430\end{array}$ & $\begin{array}{l}1,480 \\
1,740 \\
2,285 \\
1,240\end{array}$ & $\begin{array}{l}34.0 \\
24.7 \\
28.0 \\
33.4\end{array}$ \\
\hline $\begin{array}{l}\mathrm{I}-1- \\
\mathrm{I}-2- \\
\mathrm{I}-3 .\end{array}$ & $\begin{array}{l}2,205 \\
1,500 \\
3,145\end{array}$ & $\begin{array}{l}3,835 \\
2,940 \\
8,830\end{array}$ & $\begin{array}{l}2,735 \\
1,625 \\
2,945\end{array}$ & $\begin{array}{l}6,290 \\
3,490 \\
9,425\end{array}$ & $\begin{array}{l}3,060 \\
1,850 \\
3,790\end{array}$ & $\begin{array}{r}2,400 \\
985 \\
905\end{array}$ & $\begin{array}{l}26.7 \\
27.7 \\
12.8\end{array}$ \\
\hline $\begin{array}{l}\mathrm{K}-1 \\
\mathrm{~K}-2\end{array}$ & $\begin{array}{l}1,155 \\
2,990\end{array}$ & $\begin{array}{l}2,405 \\
7,460\end{array}$ & $\begin{array}{l}1,155 \\
2,480\end{array}$ & $\begin{array}{l}2,655 \\
7,785\end{array}$ & $\begin{array}{l}1,030 \\
2,970\end{array}$ & $\begin{array}{l}1,310 \\
1,070\end{array}$ & $\begin{array}{l}30.1 \\
17.0\end{array}$ \\
\hline $\begin{array}{l}M-1 \\
M-2\end{array}$ & $\begin{array}{r}850 \\
2,895\end{array}$ & $\begin{array}{l}2,040 \\
6,435\end{array}$ & $\begin{array}{r}830 \\
2,515\end{array}$ & $\begin{array}{l}2,335 \\
6,325\end{array}$ & $\begin{array}{l}1,140 \\
3,080\end{array}$ & $\begin{array}{l}790 \\
890\end{array}$ & $\begin{array}{l}30.2 \\
20.3\end{array}$ \\
\hline Q-1_. & 2,685 & 5,885 & 2,355 & 5,870 & 3,170 & 1,020 & 22.0 \\
\hline $\begin{array}{l}\text { R-1 } \\
\text { R-2 } \\
\text { R-3 }\end{array}$ & $\begin{array}{r}3,210 \\
1,865 \\
475\end{array}$ & $\begin{array}{l}7,655 \\
4,065 \\
465\end{array}$ & $\begin{array}{r}3,090 \\
1,815 \\
575\end{array}$ & $\begin{array}{l}8,960 \\
4,300 \\
875\end{array}$ & $\begin{array}{l}3,450 \\
2,030 \\
750\end{array}$ & $\begin{array}{r}1,375 \\
2,300 \\
670\end{array}$ & $\begin{array}{l}17.2 \\
30.6 \\
37.9\end{array}$ \\
\hline $\mathrm{T}-1$ & 2,230 & 3,590 & 2,490 & 5,345 & 3,140 & 2,790 & 25.7 \\
\hline
\end{tabular}

\section{PYROMETRIC CONE EQUIVALENTS}

The P. C. E. values for all the materials included in this study are given in Table 1 and, with the exception of samples $\mathrm{B}-3$ and $\mathrm{C}-3$ (Pennsylvania plastic fire clays), have a P. C. E. value of 30 or above.

\section{LINEAR THERMAL EXPANSION}

The linear thermal expansions of the 26 fire-clay bodies were determined from atmospheric temperature to $1,000^{\circ} \mathrm{C}$. Determinations were made on the clays after having been fired at $1,155^{\circ} \mathrm{C}$. and also at $1,400^{\circ} \mathrm{C}$. Check expansion determinations were made of all specimens, and the greatest variation in the data obtained on the same body was less than \pm 2.5 per cent.

The average coefficients of linear expansion for several different temperature ranges are given in Table 7 . These values are given to show how misleading it may be to accept the average coefficient of expansion from $20^{\circ}$ to $1,000^{\circ} \mathrm{C}$. as expressing the true thermal expan- 
sicn behavior of the clay on heating, since it gives no indication of the volume changes which may occur over short temperature ranges. For example, the coefficient of expansion from $20^{\circ}$ to $1,000^{\circ} \mathrm{C}$. for clay B-2 gives no intimation that the material expands much more rapidly between $100^{\circ}$ and $200^{\circ} \mathrm{C}$. (fig. 4) than between $200^{\circ}$ and $1,000^{\circ}$ C. The data given show the effect on the thermal expansion of firing the clays at two widely different temperatures and also how great the differences in this property may be over certain temperature ranges.

TABLE 7.-Coefficient of linear thermal expansion of fire clays fired at two differcnt temperatures

\begin{tabular}{|c|c|c|c|c|c|c|c|}
\hline \multirow{4}{*}{ Laboratory key letter } & \multicolumn{6}{|c|}{ A verage coefficient of expansion ${ }^{1}$ for temperature range $\left({ }^{\circ} \mathrm{C}.\right)$} & \multirow{4}{*}{$\begin{array}{c}\text { Classed } \\
\text { in } \\
\text { group } 2\end{array}$} \\
\hline & 100 & & & & $20-$ & & \\
\hline & \multicolumn{6}{|c|}{ Clays fired at $\left({ }^{\circ} \mathrm{C}.\right)-$} & \\
\hline & 1,155 & 1,400 & 1,155 & 1,400 & 1,155 & 1,400 & \\
\hline $\begin{array}{l}A-1 \\
A-2 \ldots\end{array}$ & $\begin{array}{l}12.6 \\
10.7\end{array}$ & $\begin{array}{r}13.2 \\
5.0\end{array}$ & $\begin{array}{l}6.5 \\
6.2\end{array}$ & $\begin{array}{l}5.6 \\
5.0\end{array}$ & $\begin{array}{l}6.3 \\
5.9\end{array}$ & $\begin{array}{l}6.3 \\
4.9\end{array}$ & $\begin{array}{l}2 \\
1\end{array}$ \\
\hline $\begin{array}{l}\text { B-1 } \\
\text { B-2 } \\
\text { B-3 }\end{array}$ & $\begin{array}{r}7.4 \\
12.5 \\
4.2\end{array}$ & $\begin{array}{r}4.2 \\
16.8 \\
3.7\end{array}$ & $\begin{array}{l}5.8 \\
5.5 \\
6.4\end{array}$ & $\begin{array}{l}4.2 \\
5.6 \\
4.4\end{array}$ & $\begin{array}{l}5.4 \\
6.1 \\
5.4\end{array}$ & $\begin{array}{l}4.3 \\
6.5 \\
4.4\end{array}$ & $\begin{array}{l}1 \\
2 \\
3\end{array}$ \\
\hline $\begin{array}{l}\text { C-1.... } \\
\text { C-2 }\end{array}$ & $\begin{array}{r}10.7 \\
5.3\end{array}$ & $\begin{array}{r}11.1 \\
3.6\end{array}$ & $\begin{array}{l}5.8 \\
5.8\end{array}$ & $\begin{array}{l}5.0 \\
4.5\end{array}$ & $\begin{array}{l}6.1 \\
5.2\end{array}$ & $\begin{array}{l}6.0 \\
4.4\end{array}$ & $\begin{array}{l}2 \\
3\end{array}$ \\
\hline $\begin{array}{l}\text { D-1... } \\
\text { E-1...- } \\
\text { G-1... }\end{array}$ & $\begin{array}{l}16.5 \\
13.0 \\
11.5\end{array}$ & $\begin{array}{l}19.8 \\
16.0 \\
18.7\end{array}$ & $\begin{array}{l}8.2 \\
7.1 \\
9.1\end{array}$ & $\begin{array}{l}6.7 \\
5.5 \\
7.5\end{array}$ & $\begin{array}{l}6.6 \\
6.2 \\
6.3\end{array}$ & $\begin{array}{l}7.4 \\
6.7 \\
6.8\end{array}$ & $\begin{array}{l}4 \\
4 \\
4\end{array}$ \\
\hline $\begin{array}{l}\mathrm{H}-1 \\
\mathrm{H}-2 \\
\mathrm{H}-5 \\
\mathrm{H}-6\end{array}$ & $\begin{array}{l}16.0 \\
10.9 \\
15.5 \\
16.0\end{array}$ & $\begin{array}{r}25.0 \\
6.3 \\
19.9 \\
28.2\end{array}$ & $\begin{array}{r}20.6 \\
6.3 \\
10.0 \\
19.7\end{array}$ & $\begin{array}{r}12.3 \\
5.2 \\
7.5 \\
10.0\end{array}$ & $\begin{array}{l}7.8 \\
5.9 \\
6.8 \\
7.5\end{array}$ & $\begin{array}{l}7.6 \\
5.2 \\
7.2 \\
7.8\end{array}$ & $\begin{array}{l}4 \\
1 \\
4 \\
4\end{array}$ \\
\hline $\begin{array}{l}I-1 \\
I-2 \\
I-3\end{array}$ & $\begin{array}{r}13.1 \\
11.1 \\
7.0\end{array}$ & $\begin{array}{r}16.1 \\
12.2 \\
3.7\end{array}$ & $\begin{array}{r}10.0 \\
5.6 \\
8.2\end{array}$ & $\begin{array}{l}7.4 \\
5.5 \\
4.6\end{array}$ & $\begin{array}{l}6.3 \\
6.1 \\
5.4\end{array}$ & $\begin{array}{l}6.6 \\
6.4 \\
4.1\end{array}$ & $\begin{array}{l}4 \\
2 \\
1\end{array}$ \\
\hline $\mathrm{K}-1$ & $\begin{array}{r}10.8 \\
5.4\end{array}$ & $\begin{array}{r}17.5 \\
3.4\end{array}$ & $\begin{array}{l}6.0 \\
6.6\end{array}$ & $\begin{array}{l}5.3 \\
4.7\end{array}$ & $\begin{array}{l}6.6 \\
5.3\end{array}$ & $\begin{array}{l}7.0 \\
4.6\end{array}$ & $\begin{array}{l}2 \\
3\end{array}$ \\
\hline $\begin{array}{l}M-1 \\
M-2 \ldots\end{array}$ & $\begin{array}{l}4.0 \\
4.0\end{array}$ & $\begin{array}{r}12.9 \\
4.2\end{array}$ & $\begin{array}{l}4.7 \\
5.1\end{array}$ & $\begin{array}{l}5.2 \\
5.0\end{array}$ & $\begin{array}{l}4.8 \\
5.1\end{array}$ & $\begin{array}{l}6.3 \\
5.0\end{array}$ & $\begin{array}{l}5 \\
5\end{array}$ \\
\hline Q-1... & 5.3 & 4.6 & 6.1 & 5.1 & 5.6 & 5.2 & 3 \\
\hline $\begin{array}{l}R-1 \\
R-2 \\
R-3\end{array}$ & $\begin{array}{r}9.6 \\
12.0 \\
8.0\end{array}$ & $\begin{array}{r}6.2 \\
17.6 \\
21.0\end{array}$ & $\begin{array}{r}6.2 \\
6.2 \\
12.7\end{array}$ & $\begin{array}{r}4.4 \\
5.9 \\
12.0\end{array}$ & $\begin{array}{l}5.8 \\
6.7 \\
5.4\end{array}$ & $\begin{array}{l}4.9 \\
6.8 \\
7.0\end{array}$ & $\begin{array}{l}1 \\
2 \\
4\end{array}$ \\
\hline $\mathrm{T}-1 \ldots$ & 21.3 & 30.0 & 11.9 & 5.6 & 8.1 & 8. 0 & 4 \\
\hline
\end{tabular}

1 Values to be multiplied by $10^{-0}$.

2 These groups correspond to those referred to in section 6, "Linear Thermal Expansion."

The various clays may be classified into five groups, according to their thermal expansion characteristics as determined by the free silica present either as quartz or in one or more of its allotropic modifications.

Group 1.-Five bodies (A-2, B-1, H-2, I-3, and R-1) after being fired at $1,155^{\circ} \mathrm{C}$., show a high rate of expansion below $200^{\circ} \mathrm{C}$. and a somewhat less and more uniform rate between approximately $200^{\circ}$ and $1,000^{\circ} \mathrm{C}$. The data indicate that any free silica present is 
probably either in the form of tridymite or cristobalite. The expansion data obtained on these clays after firing at the higher temperature showed that the rate of expansion was practically uniform throughout the entire temperature range, indicating that the cristobalite had been converted to a highly siliceous glass. Body I-3, after the firing at $1,155^{\circ} \mathrm{C}$., in addition to the expansion characteristics described, shows also an increased rate of expansion between $500^{\circ}$ and $600^{\circ} \mathrm{C}$. caused by the inversion of the alpha to beta quartz. After firing at $1,400^{\circ} \mathrm{C}$. this body shows a slight increase in the rate between approximately $750^{\circ}$ and $850^{\circ} \mathrm{C}$. Examples of the expansion characteristics of this group are given in Figure 3 and represent the data obtained on specimens of bodies $\mathrm{H}-2$ and $\mathrm{I}-3$.
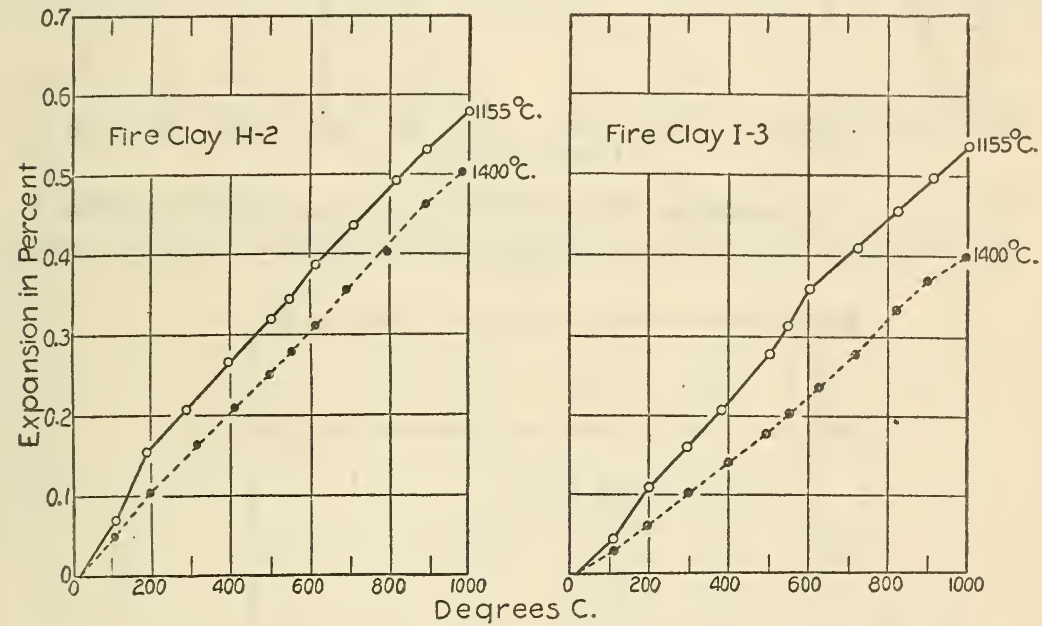

FIGURE 3.-The expansion characteristics of fire clays classed in Group 1

The curves show that the thermal expansion of these clays decreases by firing at the higher temperature

Group 2.-Six clays (B-2, I-2, K-1, R-2, A-1, and C-1) in this group are characterized by a high rate of expansion below $200^{\circ} \mathrm{C}$. occurring not only in test specimens fired at $1,155^{\circ} \mathrm{C}$., but also after having been fired at $1,400^{\circ} \mathrm{C}$. The first four clays show a higher expansion after the $1,400^{\circ} \mathrm{C}$. firing when compared with that obtained on the specimens fired at $1,155^{\circ} \mathrm{C}$. The increased total expansion is caused by the increased rate of expansion below $200^{\circ} \mathrm{C}$., as shown by the greater inflection of the curve below that temperature.

The expansions of the other two clays, namely, $\mathrm{A}-1$ and $\mathrm{C}-1$, were practically unchanged by the firing at the higher temperature. The expansion data indicate that any free silica present in these fired clays occurs as either tridymite or cristobalite. The expansions are illustrated by the curves, given in Figure 4, representing data obtained on clays B-2 and $\mathrm{C}-1$.

Group 3.-Four clays (B-3, C-2, $\mathrm{K}-2$, and $\mathrm{Q}-1)$ after the firings at both $1,155^{\circ}$ and $1,400^{\circ} \mathrm{C}$. have a comparatively uniform rate of expansion from room temperature to $1,000^{\circ} \mathrm{C}$., with the exception of a slight increase in the rate between approximately $750^{\circ}$ and $850^{\circ} \mathrm{C}$. of the clay specimens fired at $1,400^{\circ} \mathrm{C}$. The test pieces 


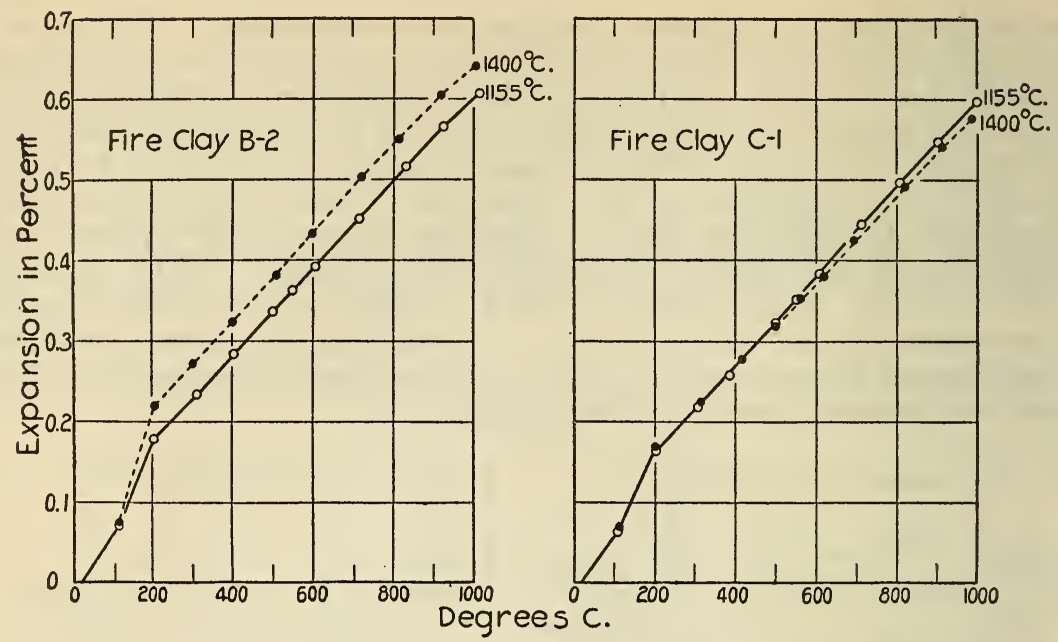

FIGURE 4.-The expansion characteristics of fire clays classed in group 2 The curves for clay B-2 show that the expansion has increased after firing at the higher temperature

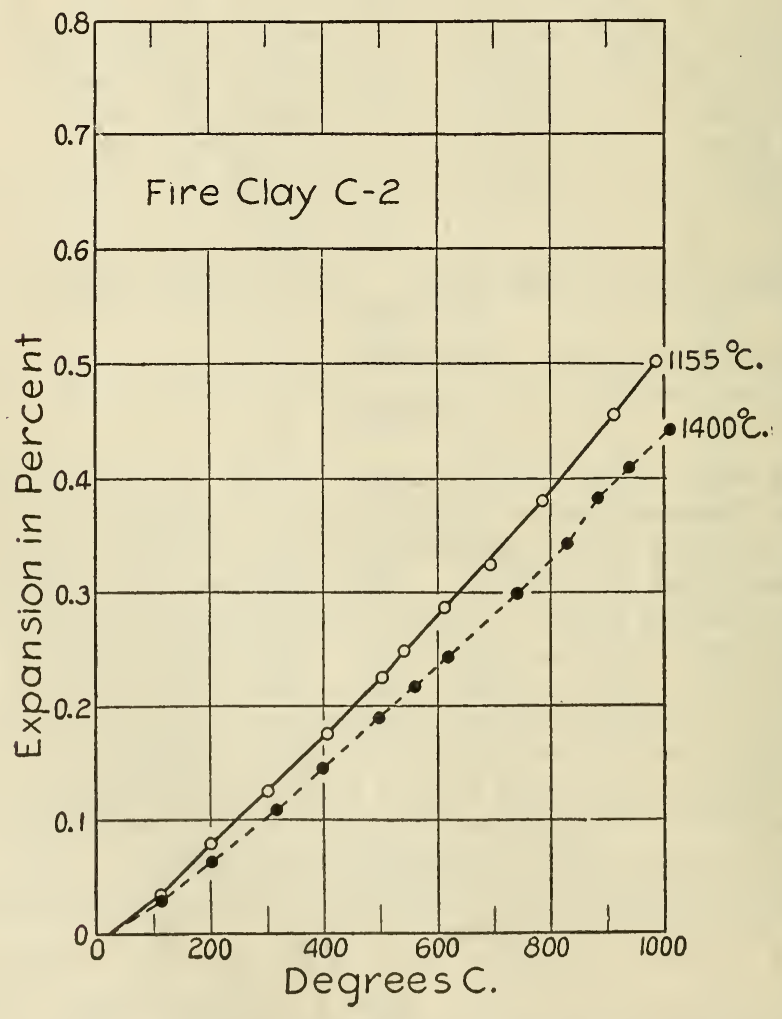

FIGURE 5.-Thermal expansion chacteristics of clays classed in group 3 are shown by the data obtained on clay $\mathrm{C}-\mathrm{Z}$ 
fired at the higher temperature show a considerably lower total expansion compared with those fired at the lower temperature. The expansion data obtained on clay $\mathrm{C}-2$ illustrating this group are shown by the curves in Figure 5.

Group 4.- -Nine clays (D-1, E-1, G-1, H-1, H-5, H-6, I-1, T-1, and $\mathrm{R}-3$ ) after firing at $1,155^{\circ} \mathrm{C}$. have a high rate of expansion below $200^{\circ} \mathrm{C}$. and also between approximately $540^{\circ}$ and $600^{\circ} \mathrm{C}$. These curves show that quartz, cristobalite, and tridymite are probably all present in the clay bodies. After firing at $1,400^{\circ} \mathrm{C}$. the inflection just below $600^{\circ} \mathrm{C}$. present in the expansion curves obtained after firing the specimens at $1,155^{\circ} \mathrm{C}$. has practically disappeared or is greatly reduced. The total expansion, however, has increased in some cases due to the conversion of the quartz into cristobalite. Although $\mathrm{R}-3$ may not strictly belong in this group because of the nature of

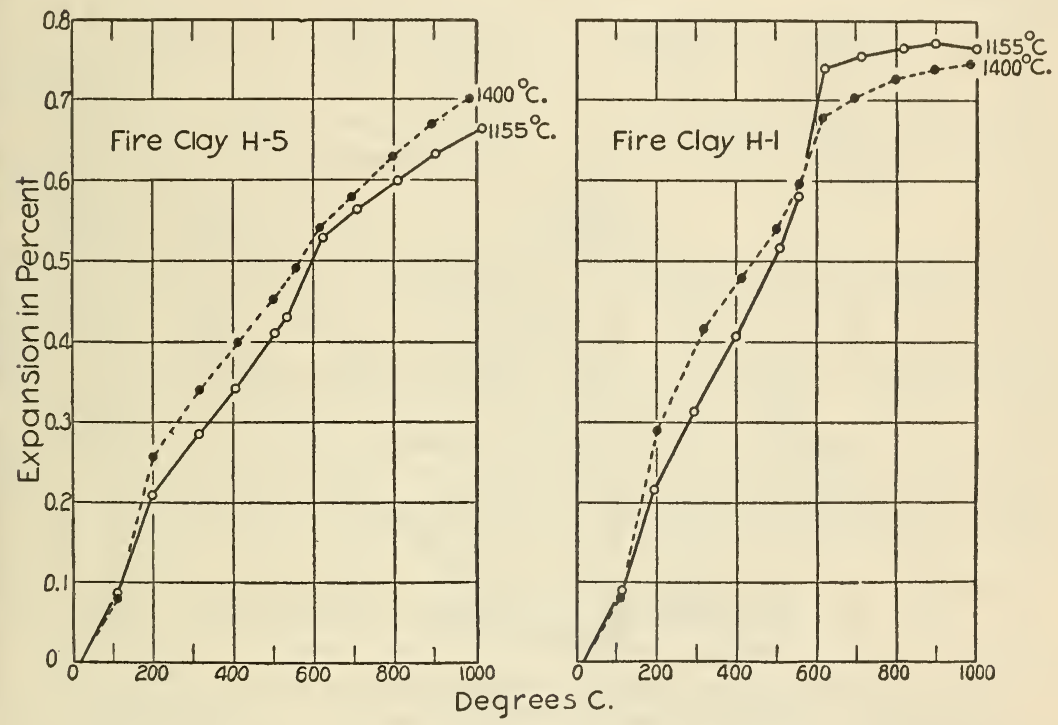

Figure 6.-The thermal expansion curves of clays $H-5$ and $H-1$ representative of clays in Group 4

The clays are of the highly siliceous type and as indicated by the curves are especially rich in cristobalite after firing at $1,400^{\circ} \mathrm{C}$.

its expansion after having been fired at $1,155^{\circ} \mathrm{C}$., it is included because the expansion shown after firing at $1,400^{\circ} \mathrm{C}$. is very similar to that of the other clays. The expansion data of clays $\mathrm{H}-1$ and $\mathrm{H}-5$, are shown by the curves in Figure 6 . The continued high rate of expansion after firing at $1,400^{\circ} \mathrm{C}$. is undoubtedly partially due to the high silica content and to the fact that the free quartz is present in comparatively coarse grains. ${ }^{11}$

Group 5.-The two rem aining bodies, $\mathrm{M}-2$ and $\mathrm{M}-1$, have individual expansion characteristics and can not readily be classed in any of the foregoing groups. Body M-2 has a uniform rate of expansion

11 Otto Bartsch calls attention to the effect of firing temperature and firing time on transformation of quartz of varying grain size in his paper Expansion Behavior of Some Quartz Containing Clays, Glastech. Ber., 3 (2), pp. 48-61; 1929 . 
from approximately $20^{\circ}$ to $1,000^{\circ} \mathrm{C}$. It is the only clay body of all those tested which shows no change in the rate of expansion after having been fired at $1,400^{\circ} \mathrm{C}$. in comparison with that obtained after the $1,155^{\circ} \mathrm{C}$. firing. The expansion curves for this body are shown in the left section of Figure 7.

The expansion data obtained on body $\mathrm{M}-1$ fired at $1,155^{\circ} \mathrm{C}$. indicate the rate to be uniform from $20^{\circ}$ to $1,000^{\circ} \mathrm{C}$. The total expansion of the material after having been fired at $1,400^{\circ} \mathrm{C}$. has increased decidedly over that obtained after having been fired at $1,155^{\circ} \mathrm{C}$. This change in total expansion is caused entirely by the high rate occurring below $200^{\circ} \mathrm{C}$., due undoubtedly to the presence of either or both cristobalite and tridymite. The probable reaction resulting in the formation of this cristobalite and tridymite is discussed under section 7. Curves for body M-1 are given on the right in Figure 7.

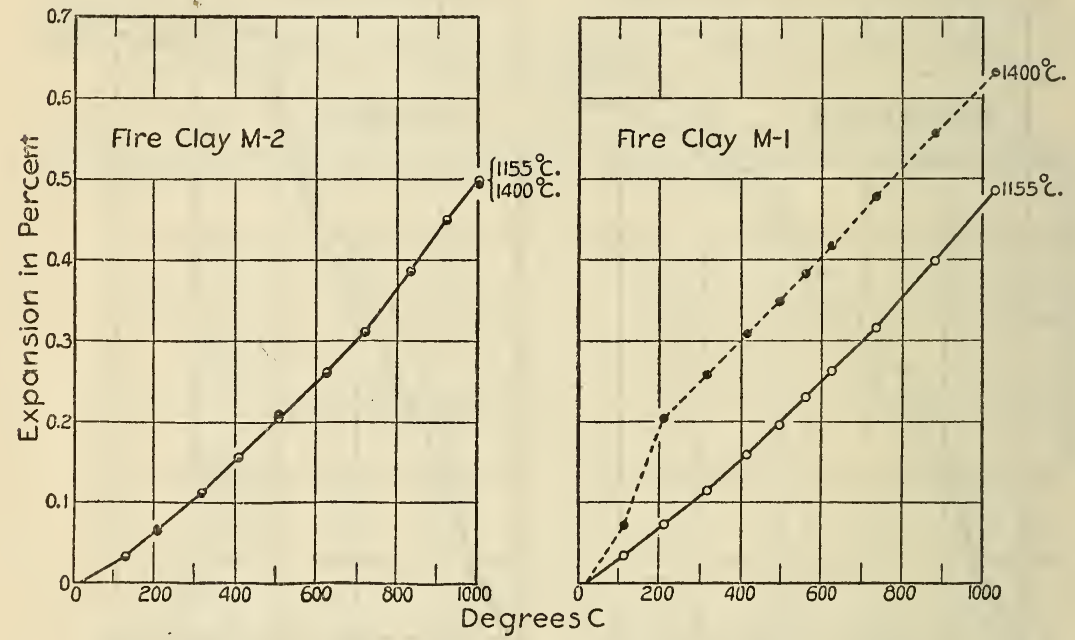

FIGURE 7.-The clays referred to in this figure have individual expansion characteristics and can not be readily classed in any of the groups illustrated

Clay M-2 apparently has the same rate of expansion whether fired at $1,155^{\circ}$ or $1,400^{\circ} \mathrm{C}$., whereas clay $M-1$ shows a decided change in this property

\section{THE EFFECT OF TEMPERATURE OF FIRING ON THE MINERALOGI- CAL COMPOSITION OF FIRE CLAYS}

It has been known for some time that mullite and free silica develop during the firing of clays in certain temperature ranges and that fluxes bear an important relation to that development. As a result of the present study it is possible to show how the linear thermal expansion is directly affected by the formation of mullite and the development of free silica accompanying it.

Five clays, namely, $\mathrm{B}-2, \mathrm{C}-1, \mathrm{I}-2, \mathrm{~K}-1$, and $\mathrm{M}-1$, whose aluminasilica ratio closely approaches that of kaolin and which contain little, if any, free quartz, were found to be especially sensitive to heat treatinent. Firing at certain temperatures caused marked changes in their mineralogical composition. This is indicated by linear thermal expansion measurements and to a lesser extent by petro- 
graphic examination. There are other clays included in this investigation whose compositions are probably affected in the same manner as the above five clays, but this particular phase of the study was limited to these five clays.

The formation of mullite from clay may be expressed as follows:

$$
3\left(\mathrm{Al}_{2} \mathrm{O}_{3} \cdot 2 \mathrm{SiO}_{2}\right)+\text { heat }=3 \mathrm{Al}_{2} \mathrm{O}_{3} \cdot 2 \mathrm{SiO}_{2}+4 \mathrm{SiO}_{2}
$$

Thermal expansion measurements were made after firing the clays at progressively increasing temperatures to show how the change in composition proceeds in accordance with the above equation. Petrographic examinations ${ }^{12}$ were made of the raw clays and also after each firing treatment.

Expansion measurements from $20^{\circ}$ to $900^{\circ} \mathrm{C}$. were made with the interferometer ${ }^{13}$ on specimens of clays $\mathrm{C}-1, \mathrm{~K}-1$, and $\mathrm{M}-1$ fired to cone 5 half down $\left(1,120^{\circ} \mathrm{C}\right.$.) ; cone $6\left(1,180^{\circ} \mathrm{C}\right.$.); cone 11 (approximately $1,260^{\circ}$ C. $)$; cone 15 half down $\left(1,400^{\circ} \mathrm{C}\right.$.); and cone $19\left(1,525^{\circ}\right.$ C.). Curres showing the data obtained on these three clays are given in Figure 8. Measurements were made on specimens of clays B-2 and I-2 only after firing at three temperatures, and data regarding them are not included.

The expansion curves (fig. 8) obtained on each of the clays after firing at $1,120^{\circ} \mathrm{C}$. show the rate of expansion to be uniform from $20^{\circ}$ to $900^{\circ} \mathrm{C}$. Expansion measurements made on the clays after firing at $1,525^{\circ} \mathrm{C}$. also show the rate of expansion to be uniform, but slightly below that obtained on specimens fired at the lower temperature in the case of $\mathrm{C}-1$ and $\mathrm{K}-1$, but decidedly lower in the case of $\mathrm{M}-1$, as may be seen from the curves in Figure 8. The changes occurring in both the mineralogical composition and thermal expansion characteristics of the materials when fired at temperatures between $1,120^{\circ}$ and $1,525^{\circ} \mathrm{C}$. are quite decided, as indicated both by the results of the petrographic examination and by the expansion curves given in Figure 8. A description of these changes follows:

Clay C-1.-Fired at $1,120^{\circ} \mathrm{C}$ - - Rate of expansion uniform from $20^{\circ}$ to $900^{\circ} \mathrm{C}$. The petrographic examination shows the clay to be wholly converted to an isotropic, probably amorphous material with a mean index of refraction of 1.57. This serves as a matrix for minute particles which are birefringent and have a very different index from the amorphous material.

Fired at $1,180^{\circ} \mathrm{C}$.- The total expansion after firing at $1,180^{\circ} \mathrm{C}$. has increased appreciably from $20^{\circ}$ to $200^{\circ} \mathrm{C}$. when compared with that obtained after firing at $1,120^{\circ} \mathrm{C}$. This change in expansion is caused by the partial formation of mullite from the clay with the liberation of silica in accordance with the above equation. The change in the rate of expansion below $200^{\circ} \mathrm{C}$. indicates that the silica which is set free (excess silica) is probably present as cristobalite.

Fired at $1,260^{\circ}$ C.-The maximum total expansion both at $200^{\circ}$ and $900^{\circ} \mathrm{C}$. was obtained after firing at $1,260^{\circ} \mathrm{C}$. At $200^{\circ} \mathrm{C}$. the total expansion has increased over 150 per cent and at $900^{\circ} \mathrm{C}$. about 35 per cent when compared with that obtained at the same temperatures on the specimen fired at $1,120^{\circ} \mathrm{C}$. From the data obtained 


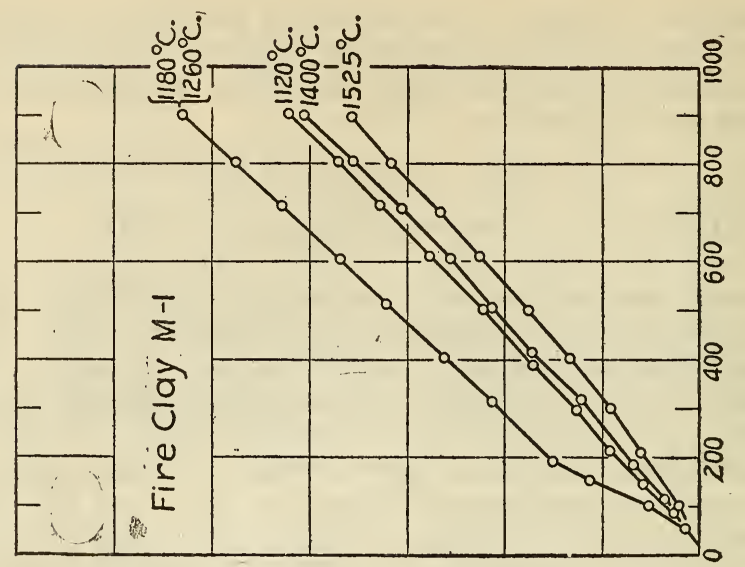

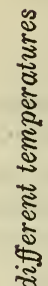

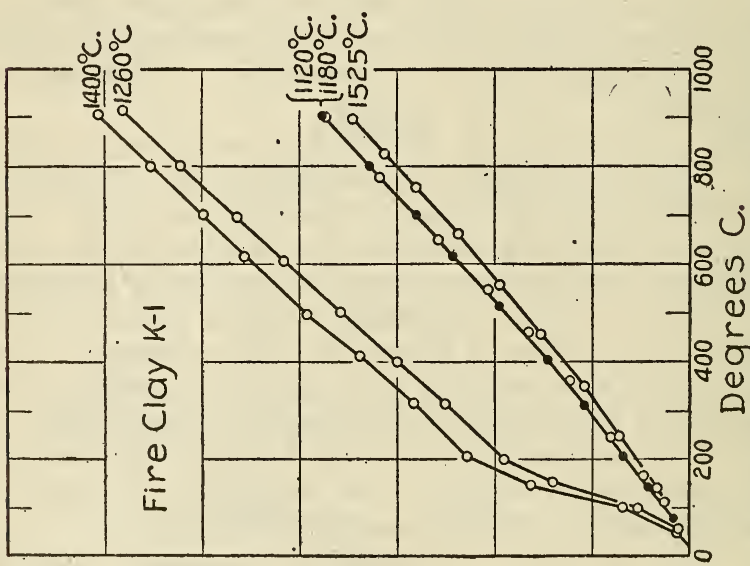

స్తి

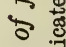

एक

8.

రิ:

है

के

ङ

$\stackrel{+}{\sigma}$

के

○

(1) 0

万)

สิ

. స్

ลี

4

अ

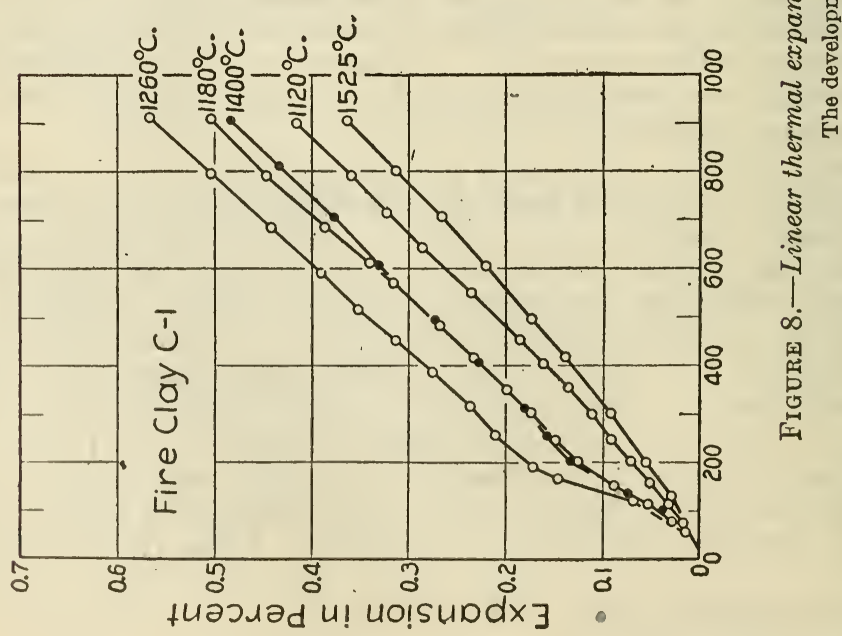


it would appear that the formation of mullite from the clay is about complete.

Fired at $1,400^{\circ}$ C.-A decided decrease in the expansion between $20^{\circ}$ and $200^{\circ} \mathrm{C}$. has taken place. The curve obtained practically coincides with that obtained after firing the clay at $1,180^{\circ} \mathrm{C}$. This indicates that the crystalline silica (cristobalite) has partially been converted to a highly siliceous glass. The petrographic examination shows only two substances present: $(a)$ Minute needles of mullite with a maximum length of about $0.012 \mathrm{~mm}$ and a maximum width of about $0.002 \mathrm{~mm}$. (b) A material which forms a matrix for the mullite and has a much lower index.

The measured mean index of refraction of this fired clay was found to be $1.582 \pm 0.003$. By calculation the mean index should be 1.579 if the grains were composed of mullite and cristobalite and 1.565 if the grains were composed of mullite and glass.

Fired at $1,525^{\circ} \mathrm{C}$.- The rate of expansion of the clay is again uniform throughout the entire range studied and the total expansion is appreciably lower than that obtained after firing at $1,120^{\circ} \mathrm{C}$., which was the lowest of any of the previous measurements. The petrographic examination shows this fired clay to be composed entirely of mullite and a glassy matrix material. The mullite needles are somewhat larger (about $0.004 \mathrm{~mm}$ in width) than those found after that firing at $1,400^{\circ} \mathrm{C}$. Mean index measurements on grains composed of mullite and the matrix material gave uncertain results. The glassy matrix material was too small in quantity to identify definitely; however, the thermal expansion measurements would indicate that the cristobalite has been wholly converted to glass.

ClaY K-1.-Fired at 1,120 C.-The petrographic examination showed an amorphous matrix in which are embedded minute particles with a greatly different index and generally high birefringence. The mean index of the grains is about 1.57. The rate of expansion is uniform throughout the entire range measured.

Fired at $1,180^{\circ} \mathrm{C}$.-The expansion curve coincides with that obtained after firing the clay at $1,120^{\circ} \mathrm{C}$. This would indicate that the formation of mullite from the clay does not occur at as low a temperature as in the case of either clay $\mathrm{C}-1$ or $\mathrm{M}-1$, although there is very little difference in the P. C. E. value of the three clays.

Fired at $1,260^{\circ}$ C.-The rate of expansion below $200^{\circ} \mathrm{C}$. and the total expansion at both $200^{\circ}$ and $900^{\circ} \mathrm{C}$. has increased greatly in comparison with that obtained after firing at either $1,120^{\circ}$ or $1,180^{\circ} \mathrm{C}$. It would appear that the formation of mullite from the clay proceeds at a very rapid rate when once the proper temperature is reached.

Fired at $1,400^{\circ}$ C.-The total expansion both at $200^{\circ}$ and $900^{\circ} \mathrm{C}$. has reached its maximum after firing the clay at this temperature At $200^{\circ} \mathrm{C}$. the total expansion is over 260 per cent greater and at $900^{\circ} \mathrm{C}$. over 60 per cent greater than that obtained at these temperatures on the specimens fired at $1,120^{\circ} \mathrm{C}$. The petrographic examination shows this fired clay to consist of mullite and a matrix material too small in quantity to indentify. An occasional small grain of quartz was observed. The thermal expansion shows that the liberated silica (cristobalite) appeared in greatest quantity during this firing.

Fired at $1,525^{\circ} \mathrm{C}$.-The rate of expansion is again uniform throughout the entire range measured and the total expansion is lower than 
that obtained on this clay after firing at any of the other temperatures. The inflection in the curve caused by the expansion of the cristobalite has entirely disappeared. The petrographic examination shows the fired clay to consist of small mullite crystals in a matrix material, the index of which it is impossible to determine. The variations which may occur in the thermal expansion of fire clays, depending on the changes in mineralogical composition caused by firing at different temperatures, are shown to unusually good advantage by the data obtained on this clay.

CLAY M-1.-Fired at $1,120^{\circ}$ C.- The petrographic examination shows that the material is composed largely of an isotropic matrix which has a slightly fibrous texture. Minute inclusions, with an index of refraction very different from the matrix and showing high birefringence, are present. The mean index of the grains is about 1.565. The fibrous texture may be an inheritance from the original platy structure of the clay. The rate of expansion is uniform from $20^{\circ}$ to $900^{\circ} \mathrm{C}$.

Fired at $1,180^{\circ}$ and $1,260^{\circ} \mathrm{C}$.- The maximum expansion both at $200^{\circ}$ and $900^{\circ} \mathrm{C}$. was reached after firing at $1,180^{\circ} \mathrm{C}$. and is identical with that obtained after firing the clay at $1,260^{\circ} \mathrm{C}$.

Fired at $1,400^{\circ} \mathrm{C}$.- The inflection in the expansion curve at $200^{\circ} \mathrm{C}$. has entirely disappeared, which is in contrast with clays $\mathrm{C}-1$ and $\mathrm{K}-1$. The petrographic examination shows that the grains are composed only of mullite distributed uniformly throughout a matrix material. Mean index of the grains is about 1.58. The formation of mullite from the clay apparently proceeds at a very rapid rate, and the reaction referred to at the beginning of this section is apparently completed much sooner than in case of either clay $\mathrm{C}-1$ or $\mathrm{K}-1$.

Fired at $1,525^{\circ}$ C.-The rate of expansion is uniform and the total expansion is lower than that obtained on the clay after the firings at any other temperature. The petrographic examination shows the grains to be composed only of mullite and a matrix material. The mullite needles are somewhat larger and better developed than in the preceding firing. It is impossible to determine the index of the matrix material accurately, but it appears to be about 1.50. It would appear that the crystalline silica (cristobalite) has been converted to glass.

The data obtained in this study show that these clays have a comparatively straight line expansion when fired at $1,120^{\circ} \mathrm{C}$. and are still essentially the same in microscopic appearance as the unfired clay. As the temperature of firing is increased, both the expansion and mineralogical composition change and development of mullite is noted. The petrographic examination of the raw clays showed little or no free quartz present. Therefore, the uncombined silica indicated by the high rate of expansion below $200^{\circ} \mathrm{C}$. for specimens fired at certain temperatures above $1,120^{\circ} \mathrm{C}$. (example, clay $\mathrm{K}-1$ $1,260^{\circ} \mathrm{C}$. fire) is believed to have been set free due to the formation of mullite from the clay matter. This excess silica probably forms, according to expansion observations, as cristobalite with possibly some tridymite and gradually changes at increased temperatures to a highly siliceous glass. The petrographic examination shows the mullite needles to be embedded in a glassy matrix which is not present in sufficient quantity to be definitely identified. 
The reaction involving the formation of mullite from clay is especially interesting, since it occurs within the range of temperature used in the firing of fire-clay refractories. It is obvious that jt may reach varying stages of completion in the refractories placed in different parts of the kiln. It follows, therefore, that the properties of the refractories taken from different parts of a kiln satting must necessarily be different depending on the state of progress of the reaction when arrested.

\section{MODULI OF ELASTICITY AND TRANSVERSE STRENGTH}

The stress (load) strain (deformation) curves, from which the moduli of elasticity were computed, were determined on specimens approximately 1 inch in cross section and 12 inches long. They were placed across a 10-inch span and loaded at the center.

The transverse strengths (moduli of rupture) of the various fire clays were computed from the loads required to break the specimens on which the load-deflection curves were determined.

The two extreme types of rupture which occurred in the testing of these clay specimens are shown in Figure 9. The upper specimen (1) shows the break to be practically perpendicular to the base of the specimen, and is associated with bodies which are open burning. The specimen (2) just below this shows a type of break which has a tendency to result in a partially curved surface. Such ruptures occur in dense or highily vitrified bodies.

Determinations of moduli of elasticity and rupture were made on specimens of the fire-clay bodies after having been fired at $1,155^{\circ} \mathrm{C}$. and also at $1,400^{\circ} \mathrm{C}$. Data were obtained at room temperature, $550^{\circ}$ and $1,000^{\circ} \mathrm{C}$., and the results for the clays fired at the lower temperature are given in Table 5 and those fired at the higher temperature in Table 6. All values, with the exception of those determined at $1,000^{\circ} \mathrm{C}$., represent the average of not less than two determinations, either value making up this average varying less than 10 per cent from that average.

Comparing the results obtained at $20^{\circ} \mathrm{C}$. on specimens fired at $1,155^{\circ} \mathrm{C}$. (Table 5) with those fired at $1,400^{\circ} \mathrm{C}$. (Table 6 ) the following information is obtained:

(a) With four exceptions, B-3, C-2, R-2, and R-3, which show decreases, the bodies show greater transverse strengths after the firing at the higher temperature. Although several of the clays show little change, the majority show decided increases ranging to 100 per cent.

(b) With two exceptions, B-3 and R-3, the moduli of elasticity of the bodies are greater after firing at $1,400^{\circ} \mathrm{C}$. than after firing at $1,155^{\circ} \mathrm{C}$. The increases range from a minimum of approximately 5 per cent to a maximum of 120 per cent.

The results obtained in the tests s.t $550^{\circ} \mathrm{C}$. and $1,000^{\circ} \mathrm{C}$. are relatively the same as those at $20^{\circ}$.

A comparison of the values obtained at $550^{\circ} \mathrm{C}$. with those obtained at $20^{\circ} \mathrm{C}$. of specimens fired at either $1,155^{\circ}$ or $1,400^{\circ} \mathrm{C}$. shows, in general, that the percentage increase in moduli of elasticity and rupture is greater in highly siliceous bodies than in aluminous bodies. 


\section{PLASTIC FLOW}

Deflection readings resulting from the plastic flow measured at $1,000^{\circ}$ C. of the 26 fire-clay bodies fired at $1,155^{\circ}$ and $1,400^{\circ}$ C. are given in Table 8, which is divided into two major parts. The left section presents data obtained on specimens of clay fired at $1,155^{\circ} \mathrm{C}$. and the right section those fired at $1,400^{\circ} \mathrm{C}$. The first two columns in each section represent the average of increments obtained every five minutes after the first five minutes for one hour when using a stress of approximately $60 \mathrm{lbs}$./in. ${ }^{2}$ and a second test for a one-half hour period when using a stress of approximately $120 \mathrm{lbs} . / \mathrm{in}^{2}{ }^{2}$ The load was applied midspan of the 1 by 1 by 12 inch specimens on a 10-inch span.

TABLE 8.-Plastic flow data measured at $1,000^{\circ} \mathrm{C}$. on fire clays

\begin{tabular}{|c|c|c|c|c|c|c|}
\hline \multirow{4}{*}{ Clay No. } & \multicolumn{6}{|c|}{ Clays fired at- } \\
\hline & \multicolumn{3}{|c|}{$1,155^{\circ} \mathrm{C}$} & \multicolumn{3}{|c|}{$1,400^{\circ} \mathrm{C}$} \\
\hline & \multicolumn{2}{|c|}{$\begin{array}{l}\text { A verage deflection } \\
\text { readings, taken at } \\
5 \text {-minute intervals, } \\
\text { on } 1 \text { by } 1 \text { by } 12 \text { inch } \\
\text { specimens using a } \\
\text { 10-inch span }\end{array}$} & \multirow{2}{*}{$\begin{array}{c}\text { Total } \\
\text { plastic } \\
\text { deflection, } \\
\text { taken from } \\
\text { plastic flow } \\
\text { curves, } \\
\text { with stress } \\
\text { approxi- } \\
\text { mately } 360 \\
\text { lbs./in. }\end{array}$} & \multicolumn{2}{|c|}{$\begin{array}{l}\text { Average deflection } \\
\text { readings, taken at } \\
5 \text {-minute intervals, } \\
\text { on } 1 \text { by } 1 \text { by } 12 \text { inch } \\
\text { specimens using a } \\
\text { 10-inch span }\end{array}$} & \multirow{2}{*}{$\begin{array}{l}\text { Total } \\
\text { plastic } \\
\text { deflection, } \\
\text { with stress } \\
\text { approxi- } \\
\text { mately } 360 \\
\text { lbs./in. } \\
\text { (taken from } \\
\text { plastic flow } \\
\text { curves; see } \\
\text { footnote } \\
\text { No. 14) }\end{array}$} \\
\hline & $\begin{array}{c}\text { During 1 } \\
\text { hour; stress } \\
\text { approxi- } \\
\text { mately } 60 \\
\text { lbs./in. }\end{array}$ & $\begin{array}{c}\text { During } 1 / 2 \\
\text { hour; stress } \\
\text { approxi- } \\
\text { mately } 120 \\
\text { lbs./in. }\end{array}$ & & $\begin{array}{c}\text { During } 1 \\
\text { hour; stress } \\
\text { approxi- } \\
\text { mately } 60 \\
\text { lbs./in.2 }\end{array}$ & $\begin{array}{l}\text { During } 1 / 2 \\
\text { hour; stress } \\
\text { approxi- } \\
\text { mately } 120 \\
\text { lbs./in.2 }\end{array}$ & \\
\hline $\begin{array}{l}A-1 \\
A-2\end{array}$ & $\begin{array}{l}\text { Inch } \\
<0.0001 \\
.0002\end{array}$ & $\begin{array}{l}\text { Inch } \\
0.0001 \\
.0004\end{array}$ & $\begin{array}{l}\text { Inch } \\
0.0025 \\
.0048\end{array}$ & $\begin{array}{l}\text { Inch } \\
<0.0001 \\
<.0001\end{array}$ & $\begin{array}{l}\text { Inch } \\
<0.0001 \\
<.0001\end{array}$ & $\begin{array}{l}\text { Inch } \\
0.0003 \\
.0004\end{array}$ \\
\hline $\begin{array}{l}\text { B-1 } \\
\text { B-2 } \\
\text { B-3 }\end{array}$ & $\begin{array}{l}.0003 \\
.0005 \\
.0053\end{array}$ & $\begin{array}{l}.0004 \\
.0013 \\
.0138\end{array}$ & $\begin{array}{l}.0063 \\
.0208 \\
.1230\end{array}$ & $\begin{array}{r}<.0001 \\
<.0001 \\
.0005\end{array}$ & $\begin{array}{r}<.0001 \\
.0001 \\
.0005\end{array}$ & $\begin{array}{l}.0007 \\
.0013 \\
.0080\end{array}$ \\
\hline C-1 -2 & $\begin{array}{l}.0004 \\
.0007\end{array}$ & $\begin{array}{l}.0020 \\
.0014\end{array}$ & $\begin{array}{l}.0156 \\
.0062\end{array}$ & $\begin{array}{l}<.0001 \\
<.0001\end{array}$ & $\begin{array}{l}.0001 \\
.0001\end{array}$ & $\begin{array}{l}.0010 \\
.0008\end{array}$ \\
\hline $\begin{array}{l}\text { D-1 } \\
\text { E-1............ } \\
\text { G-1 }\end{array}$ & $\begin{array}{l}.0003 \\
.0001 \\
.0001\end{array}$ & $\begin{array}{l}.0004 \\
.0002 \\
.0002\end{array}$ & $\begin{array}{l}.0018 \\
.0018 \\
.0018\end{array}$ & $\begin{array}{l}<.0001 \\
<.0001 \\
<.0001\end{array}$ & $\begin{array}{c}<.0001 \\
<.0001\end{array}$ & $\begin{array}{l}.0003 \\
.0003 \\
.0006\end{array}$ \\
\hline $\begin{array}{l}\mathrm{H}-1-2 . \\
\mathrm{H}-2 \\
\mathrm{H}-5-6\end{array}$ & $\begin{array}{r}.0001 \\
.0001 \\
<.0001 \\
.0003\end{array}$ & $\begin{array}{l}.0003 \\
.0001 \\
.0002 \\
.0004\end{array}$ & $\begin{array}{l}.0060 \\
.0022 \\
.0020 \\
.0050\end{array}$ & $\begin{array}{r}<.0001 \\
<.0001 \\
<.0001 \\
.0001\end{array}$ & $\begin{array}{r}<.0001 \\
<.0001 \\
<.0001 \\
.0001\end{array}$ & $\begin{array}{l}.0004 \\
.0009 \\
.0002 \\
.0004\end{array}$ \\
\hline $\begin{array}{l}\text { I-1 } \\
\text { I-2 } 2 \ldots \\
\text { I-3 }-\end{array}$ & $\begin{array}{l}.0002 \\
.0005 \\
.\end{array}$ & $\begin{array}{l}.0003 \\
.0011 \\
.0004\end{array}$ & $\begin{array}{l}.0048 \\
.0275 \\
.0030\end{array}$ & $\begin{array}{r}<.0001 \\
<.0001 \\
.0002\end{array}$ & $\begin{array}{r}<.0001 \\
<.0001 \\
.0002\end{array}$ & $\begin{array}{l}.0002 \\
.0005 \\
.0018\end{array}$ \\
\hline $\begin{array}{l}\mathbf{K}-1 \ldots \\
\mathbf{K}-2 \ldots\end{array}$ & $\begin{array}{l}.0001 \\
.0005\end{array}$ & $\begin{array}{l}.0003 \\
.0006\end{array}$ & $\begin{array}{l}.0044 \\
.0072\end{array}$ & $\begin{array}{l}<.0001 \\
<.0001\end{array}$ & $\begin{array}{r}<.0001 \\
.0004\end{array}$ & $\begin{array}{l}.0010 \\
.0003\end{array}$ \\
\hline $\begin{array}{l}M-1 \\
M-2\end{array}$ & $\begin{array}{l}.0004 \\
.0003\end{array}$ & $\begin{array}{l}.0010 \\
.0004\end{array}$ & $\begin{array}{l}.0184 \\
.0056\end{array}$ & $\begin{array}{l}<.0001 \\
<.0001\end{array}$ & $\begin{array}{l}<.0001 \\
<.0001\end{array}$ & $\begin{array}{l}.0011 \\
.0006\end{array}$ \\
\hline Q-1.-. & .0002 & .0008 & .0036 & $<.0001$ & $<.0001$ & .0007 \\
\hline $\begin{array}{l}R-1 \\
R-2 \\
R-3\end{array}$ & $\begin{array}{r}<.0001 \\
<.0001 \\
.0002\end{array}$ & $\begin{array}{l}.0003 \\
.0002 \\
.0002\end{array}$ & $\begin{array}{l}.0032 \\
.0030 \\
.0036\end{array}$ & $\begin{array}{r}.0001 \\
<.0001 \\
<.0001\end{array}$ & $\begin{array}{r}.0001 \\
.0001 \\
<.0001\end{array}$ & $\begin{array}{l}.0010 \\
.0003 \\
.0017\end{array}$ \\
\hline$T-1 \ldots$ & $<.0001$ & .0002 & .0016 & $<.0001$ & $<.0001$ & .0004 \\
\hline
\end{tabular}




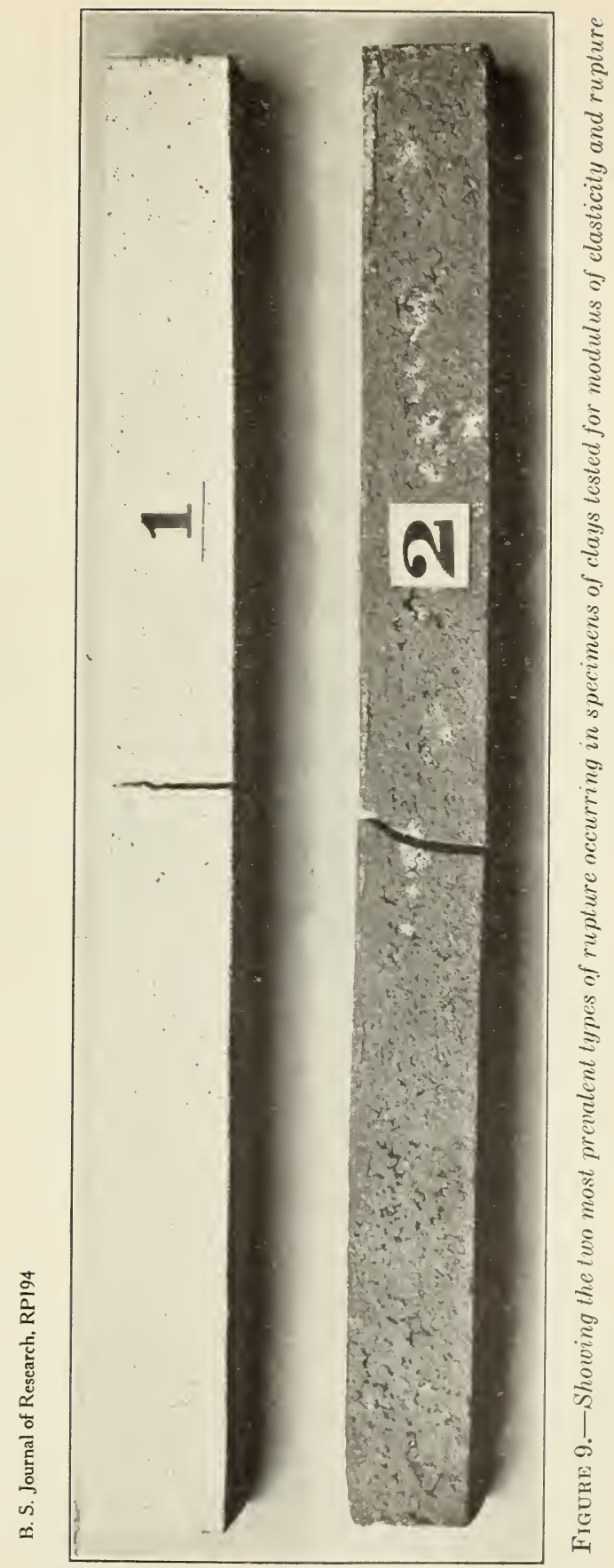




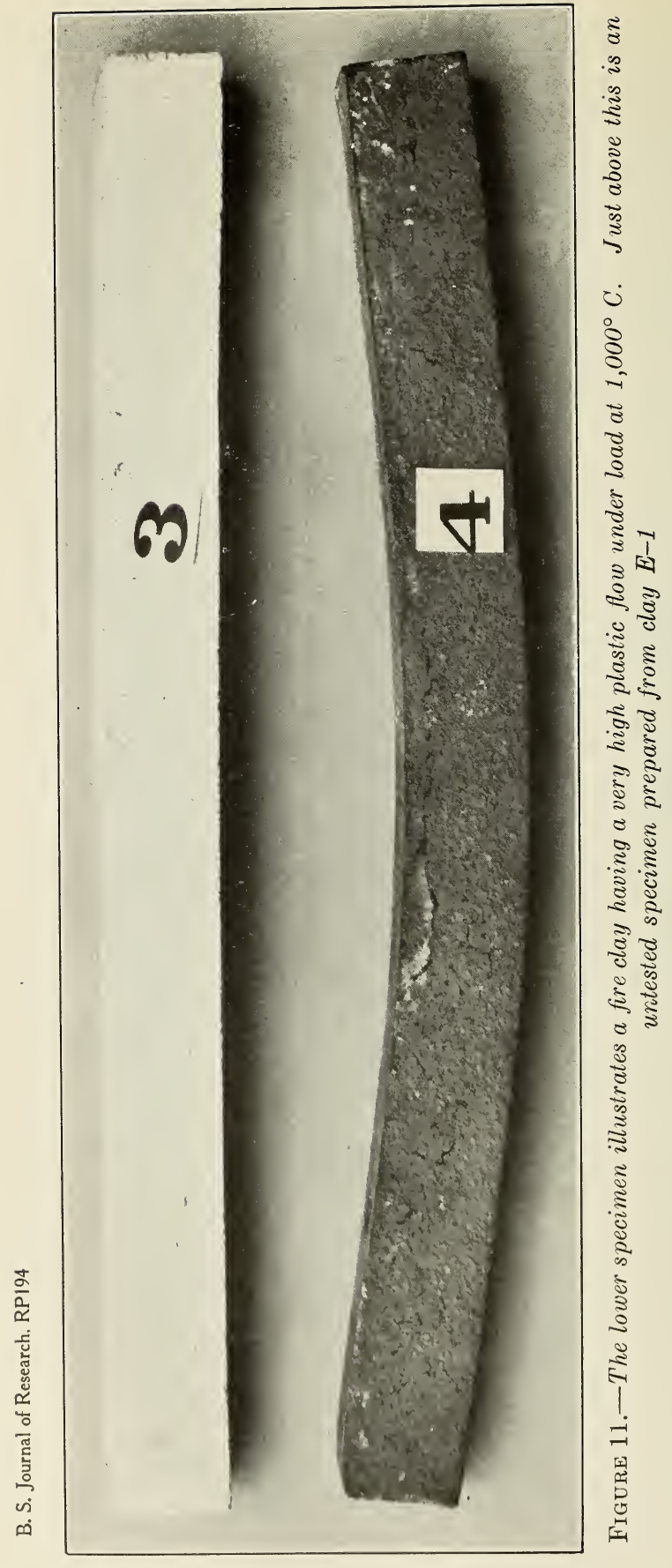


The data obtained with these loads applied to clays B-3 and M-1, which represent two clays of widely different plastic-flow properties, are shown in Figure 10. These curves illustrate the effect of temperature of firing on the plastic flow as well as the relative amounts which take place after the first 5-minute interval. A large part of the deflection taking place during the first-time interval represents the elastic properties of the clay. In the case of clay $\mathbf{M}-1$, fired at

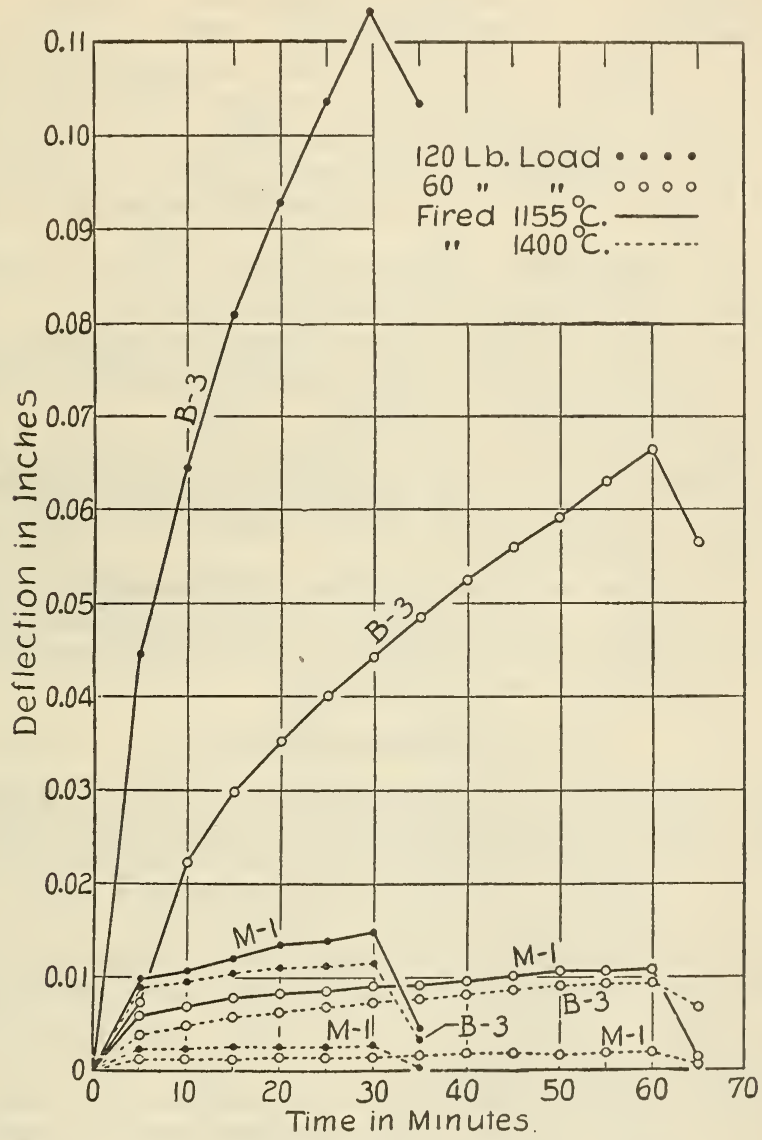

Figure 10.-Time-deflection curves obtained at $1,000^{\circ}$ $C$. on clays $B-3$ and $M-1$

Constant loads of approximately 60 and $120 \mathrm{lbs}$./in. ${ }^{2}$ were applied for periods of one hour and one-half hour, respectively. As indicated by the curve the final reading was taken after the removal of the load

$1,400^{\circ} \mathrm{C}$., there is very little plastic flow after the initial time increment. This is in contrast with clay B-3, which shows continued flow to the final reading.

The values given in the third column of each section of Table 8 represent the total plastic deflection in inches of the various clays under a stress of approximately $360 \mathrm{lbs} . / \mathrm{in} .{ }^{2}$. The values were taken from the plastic-flow curves described as follows in the Progress 
Report on Investigation of Fire-Clay Bricks and the Clays Used in Their Preparation: ${ }^{14}$

Typical load-deflection curves as obtained at $1,000^{\circ} \mathrm{C}$. on a specimen cut from fire-clay brick are shown in Figure 13. The upper curve shows the plastic flow of the material under these same loads and the middle curve the elastic properties. The elastic recovery of the material was considered as the difference between the total defiection caused by the load and the "set" ("representing plastic flow") of the material on removal of this load.

The results of this study may be summarized as follows:

1. The plastic flow of these clays fired at either $1,155^{\circ}$ or $1,400^{\circ} \mathrm{C}$. when loads of $60 \mathrm{lbs} . / \mathrm{in} .^{2}$ and $120 \mathrm{lbs} . / \mathrm{in} .^{2}$ are applied is very small.

2. The values of plastic deflection are decidedly different for the various clays when loads greater than those referred to under (1) are used. This is indicated by the results given in Table 8 when a load of $360 \mathrm{lbs} . /$ in. $^{2}$ is used, and is especially apparent in the clays fired at $1,155^{\circ} \mathrm{C}$.

3. The plastic deflections of the clays fired at $1,400^{\circ} \mathrm{C}$. are decidedly lower than those of the same clays fired at $1,155^{\circ} \mathrm{C}$.

4. The highly siliceous clays ${ }^{15}$ show the least plastic deflection after firing either at $1,155^{\circ}$ or $1,400^{\circ} \mathrm{C}$. Next in order are the siliceous clays, which are followed by aluminous.

5. The plastic deflection of the clays after firing at $1,400^{\circ} \mathrm{C}$. is not greatly different. It would appear that the development of the crystal phase in the aluminous clays is decidedly advantageous in decreasing their plastic flow.

Figure 11 shows an untested specimen (3) in contrast with one (4) that has been tested. Specimen 4, which is prepared from clay B-3, deflected beyond the capacity of the testing apparatus without rupturing and represents an extreme case.

\section{GROUPING OF CLAYS}

With the exception of three samples of silica or quartz $(\mathrm{H}-3$, $\mathrm{H}-4$, and $\mathrm{P}-1$ ), all are plotted according to chemical analyses on the small section of a triaxial diagram shown in Figure 12. The values given for silica, alumina, and fiux are computed to a water-free basis. Since all of the compositions fall in a small section of the diagram, it was considered desirable to show only that section on an enlarged scale. The lower left apex of the section represents 55 per cent alumina, the apex at the right 35 per cent flux, and that at the top 80 per cent silica. The position in the diagram indicated by the cross within the circle represents the composition of a washed North Carolina kaolin, and is given simply to show how the various clays compare in composition to typical kaolin.

The clays have been divided into three groups, as indicated by the dashed lines. The maximum percentage of mullite, which may theoretically be obtained from a clay of given composition fired under favorable conditions for such development, was computed from the chemical analysis (water-free basis). These values are only approximate since, according to the nature of the system, some of the alumina must necessarily have gone into solution with the glass which is present. The line $Y Y^{\prime}$ was so placed as to include those clays (Group A) having less than 40 per cent "free silica" after the formation of mullite. The area between lines $Y Y^{\prime}$ and $Z Z^{\prime}$ inciudes those (Group B) which

14 I3. S. Jour. Research, 3 (IR P114). Also J. Am. Ceram. Soc., 12 (10), pp. 640-675; 1929.

16 Arbitrarily considered as those containing more than 60 per cent silica (water-free basis). 
theoretically contain between 40 and 50 per cent "free silica" and those falling above line $Z Z^{\prime}$ (Group C) contain over 50 per cent. Important properties of the three groups are indicated by the values given in the following table:

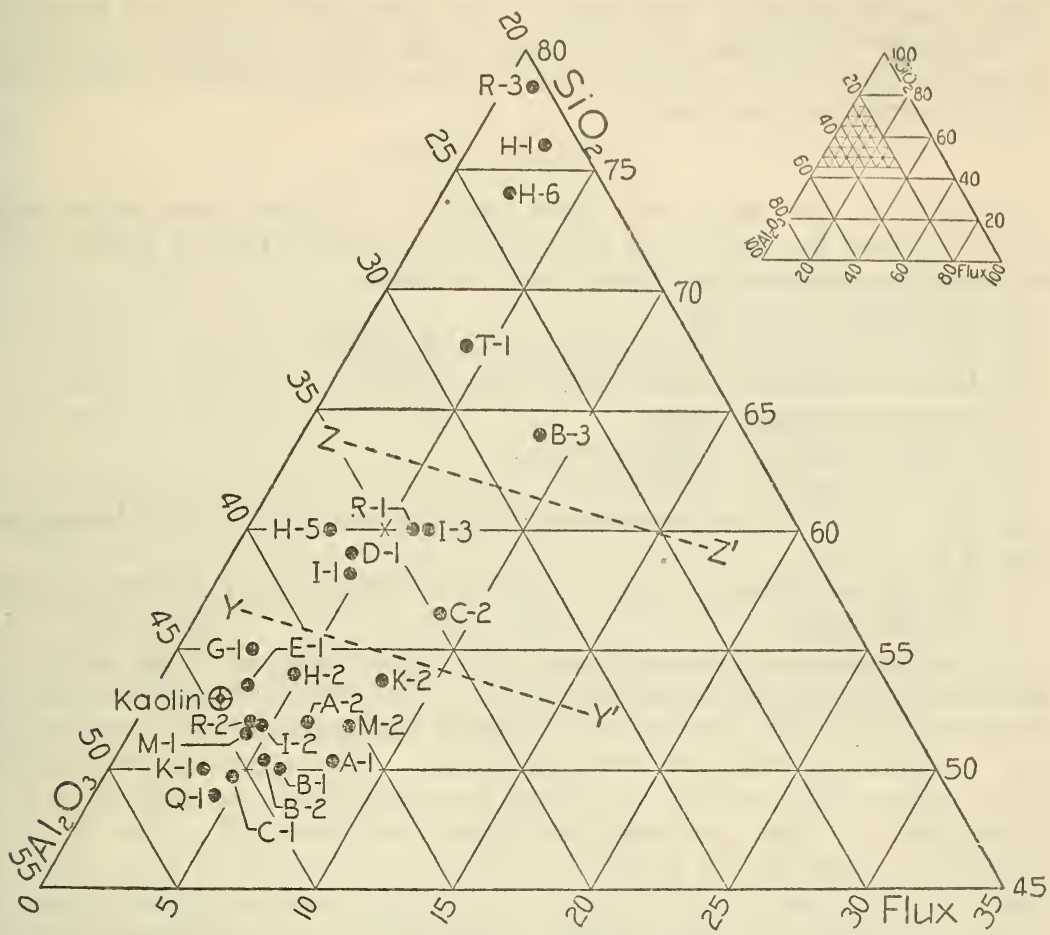

FIGURE 12.-A seciion of a triaxial diagram (the shadea portion enlarged of the small triangle on the right) showing the location of clays according to to chemical analysis

A kaolin is included for comparative purposes only.

These values show the percentage increase in properties for specimens fired at $1,400^{\circ} \mathrm{C}$. as compared with those fired at $1,155^{\circ} \mathrm{C}$. and are given as average percentages for each group

\begin{tabular}{|c|c|c|c|c|}
\hline & Porosity & $\begin{array}{l}\text { Modulus of } \\
\text { rupture } 1 \\
\text { at } 20^{\circ} \mathrm{C} \text {. }\end{array}$ & $\begin{array}{l}\text { Modulus of } \\
\text { elasticity } \\
\text { at } 20^{\circ} \mathrm{C} \text {. }\end{array}$ & $\begin{array}{l}\text { Modulus of } \\
\text { clasticity } \\
\text { at } 1,000^{\circ} \mathrm{C} \text {. }\end{array}$ \\
\hline $\begin{array}{l}\text { Group A (less than } 40 \text { per cent }{ }^{2} \text { free } \mathrm{SiO}_{2} \text { ) } \\
\text { Group } \mathrm{B} \text { (between } 40 \text { and } 50 \text { per cent free } \mathrm{SiO}_{2} \text { ) } \\
\text { Group } \mathrm{C}^{3}\end{array}$ & $\begin{array}{r}-10.8 \\
-30.6 \\
-8.8\end{array}$ & $\begin{array}{l}30.8 \\
50.9 \\
25.0\end{array}$ & $\begin{array}{l}44.4 \\
71.6 \\
37.9\end{array}$ & $\begin{array}{l}133.3 \\
180.7 \\
120.3\end{array}$ \\
\hline
\end{tabular}

1 Determinations for this purpose made on a 4-inch span because of greater number available.

2 Refers to "free silica" which could theoretically be present after the formation of muilite.

3 Clay B-3 not considered in group average because of its low P. C. E. value.

These averages indicate that the clays classed in grouns $\mathrm{A}$ and $\mathrm{C}$ have properties which are probably more desirable in clays for the manufacture of refractories than those classed in group B. The percentage change in porosity would indicate that the firing range of clays in group $B$ is shorter than that of the clays included in the other groups. The percentage change in values for moduli of elesticity, both at $20^{\circ}$ and $1,000^{\circ} \mathrm{C}$., and moduli of rupture show that the percentage of glass in the clays of group B is probably greater or formed 
more rapidly at the higher temperature of firing than in groups $A$ and $\mathrm{C}$. However, the average total linear thermal expansion (Table 7) at $1,000^{\circ} \mathrm{C}$. of clays in group $\mathrm{C}$ is almost 30 per cent greater than that of either of the other two groups. The high expansion of the clays in this group (see expansion curve for clay $\mathrm{H}-1$, fig. 6 ) is caused by the high percentage of quartz or silica they contain. In general, therefore, the clays in group A have properties which are most desirable in refractory products.

\section{SUMMARY}

Twenty-six fire clays and three samples of quartz and silica sand were furnished for this study by manufacturers of fire-clay refractories. Information obtained on these clays included:

1. Chemical analyses.

2. Tempering water and absorption of grog.

3. Linear shrinkage and unfired strength.

4. Porosity of fired clays.

5. Pyrometric cone equivalents.

6. Linear thermal expansion.

7. The effect of temperature of firing on the mineralogical composition of fire clays.

8. Moduli of elasticity and transverse strength of the fired clays.

9. Plastic flow.

The chemical analyses of the clays show them to range from the aluminous type to the highly siliceous. The results of such determinations as absorption, porosity, shrinkage, and pyrometric cone equivalent values are given in a series of tables. The approximate location of the mines from which the clays were obtained, as well as a brief description of their geologic formation are included. The thermal expansions from $20^{\circ}$ to $1,000^{\circ} \mathrm{C}$. of the fire clays fired at $1,155^{\circ}$ and $1,400^{\circ} \mathrm{C}$. are given, and the clays are grouped according to the several types of expansion. The moduli of elasticity and transverse breaking strengths have been determined after receiving two different heat treatments, and at several different temperatures.

These data show that the thermal expansions of the fire clays determined after firing at $1,400^{\circ} \mathrm{C}$. may be greater or less than after firing them at $1,155^{\circ} \mathrm{C}$., depending entirely on the composition of the clays. The information given also indicates the successive simultaneous development of cristobalite and mullite, and finally the probable conversion of the cristobalite to a highly siliceous glass.

The moduli of elasticity and rupture of the clays are, with few exceptions, greater after firing at $1,400^{\circ} \mathrm{C}$. than after firing at $1,155^{\circ} \mathrm{C}$.

Comparing the values for moduli of rupture and elasticity obtained at $550^{\circ} \mathrm{C}$. with those obtained at atmospheric temperature show, in general, that the percentage increase in moduli of elasticity and rupture is greater in highly siliceous clays than in aluminous clays. The plastic deflections of the clays, measured at $1,000^{\circ} \mathrm{C}$. are much lower for those fired at $1,400^{\circ} \mathrm{C}$. than those fired at $1,155^{\circ} \mathrm{C}$., and are also lower in the siliceous type of clays than in the aluminous.

The clays are plotted according to chemical composition on a triaxial diagram and are divided into three groups. Those clays which on firing would theoretically contain the greatest percentage of mullite are grouped about a kaolin and have properties which should be very desirable in fire-clay products.

Washington, February 25, 1930. 